\title{
Bayesian group belief
}

\author{
Franz Dietrich
}

Received: 8 July 2009 / Accepted: 1 April 2010 / Published online: 29 April 2010

(C) The Author(s) 2010. This article is published with open access at Springerlink.com

\begin{abstract}
If a group is modelled as a single Bayesian agent, what should its beliefs be? I propose an axiomatic model that connects group beliefs to beliefs of the group members. The group members may have different information, different prior beliefs and even different domains (algebras) within which they hold beliefs, accounting for differences in awareness and conceptualisation. As is shown, group beliefs can incorporate all information spread across individuals without individuals having to explicitly communicate their information (that may be too complex or personal to describe, or not describable in principle in the language). The group beliefs derived here take a simple multiplicative form if people's information is independent (and a more complex form if information overlaps arbitrarily). This form contrasts with familiar linear or geometric opinion pooling and the (Pareto) requirement of respecting unanimous beliefs.
\end{abstract}

\section{Introduction}

Suppose a group is interested in whether a given hypothesis $H$ is true. If every individual assigns a probability of $70 \%$ to $H$, what probability should the group as a whole assign to $H$ ? Is it exactly $70 \%$, or perhaps more since different persons have independently confirmed $H$ ? The answer, I will show, crucially depends on the informational

\footnotetext{
F. Dietrich $(\varangle)$

Department of Philosophy, Logic and Scientific Method, London School of Economics, Houghton Street, London WC2A 2AE, UK

e-mail: f.dietrich@1se.ac.uk

URL: www.franzdietrich.net

F. Dietrich

Department of Quantitative Economics, Maastricht University, Tongersestraat 53,

6211 LM Maastricht, The Netherlands
} 
states of the individuals. If they have identical information, the collective has good reasons to adopt people's unanimous $70 \%$ belief, following the popular (probabilistic) Pareto principle (e.g. Mongin 1995, 1998). Under informational asymmetry, by contrast, a possibly much higher or lower collective probability may be appropriate, and the Pareto principle becomes problematic, or so I argue.

The above question is an instance of the classic opinion pooling/aggregation problem, with applications for instance in expert panels. In general, the beliefs of different individuals need of course not coincide, and also more than one hypothesis may be under consideration. The general goal is to merge many individuals' probability assignments to certain (exclusive and exhaustive) hypotheses into a single collective probability assignment to these hypotheses. The literature has proposed different normative conditions on the aggregation rule, and has derived the class of rules satisfying these conditions. The two most prominent types of rules are linear and geometric rules. Denoting by $\pi_{1}, \ldots, \pi_{n}$ and $\pi$ the individual and collective probability assignments (each assignment being a function that maps hypotheses to probabilities), a linear rule defines $\pi$ as being a weighted arithmetic average $\sum_{i=1}^{n} w_{i} \pi_{i}$, and a geometric rule defines $\pi$ as being proportional to a weighted geometric average $\prod_{i=1}^{n} \pi_{i}^{w_{i}}$, where $w_{1}, \ldots, w_{n} \in[0,1]$ are fixed weights with sum 1 . By contrast, our Bayesian axioms will lead to what I call multiplicative rules, which define $\pi$ as $g \prod_{i=1}^{n} \pi_{i}$, the product of all (unweighted) individual function $\pi_{i}$ with some fixed function $g$. Linear rules have been characterised (under additional technical assumptions) by the independence or setwise function property (McConway 1981; Wagner 1982, 1985; Dietrich and List 2007; see also Lehrer and Wagner 1981), the marginalisation property (McConway (1981)), and (in a single-profile context) by the probabilistic analogue of the Pareto principle (Mongin 1995, 1998); and geometric rules famously satisfy external Bayesianity as defined in Sect. 6 (e.g. McConway 1978; Genest 1984; Genest et al. 1986). Still an excellent reference for fundamental results on opinion pooling is Genest and Zidek's (1986) literature review.

I claim that the classic approach is problematic if, as in this paper, the goal of opinion pooling is taken to be information aggregation, i.e. if collective beliefs should incorporate all the information spread asymmetrically over the individuals. The classic approach is more suitable if the goal is not information aggregation: the goal might be not epistemic at all (e.g. fair representation), or it might be epistemic yet with the disagreements between individuals caused not by differences in information but by differences in interpretation of the same shared body of information.

One might at first suspect that classic pooling functions can account for informational asymmetries by putting more weight on the beliefs of well-informed individuals. More concretely, it is often suggested that in a linear and geometric rule (as defined above) the weights $w_{i}$ of well-informed individuals should be higher. However, as Genest and Zidek (1986) put it, 'expert weights do allow for some discrimination [...], but in vague, somewhat ill defined ways' (p. 120), and 'no definite indications can be given concerning the choice or interpretation of the weights' (p. 118).

To concretely illustrate the difficulty that classic pooling functions have in aggregating information, consider again the introductory example. Suppose each individual $i$ 's subjective probability $\pi_{i}(H)=0.7$ is in fact the result of Bayesian conditioning on some private information. What should the collective belief $\pi(H)$ be? If the 
individuals started from the same prior probability of $H$, all depends on how this prior compares to 0.7 : if the prior is below 0.7 , say 0.5 , then $\pi(H)$ should intuitively exceed 0.7 because $\pi(H)$ should incorporate the pooled information of many individuals, where a single individual's information already suffices to push the probability of $H$ up from a prior of 0.5 to a posterior of 0.7 . By a similar argument, if $H$ has a common prior above 0.7 then intuitively $\pi(H)<0.7$, and if $H$ has a common prior of exactly 0.7 then intuitively $\pi(H)=0.7$. If people hold different prior beliefs of $H$, some below 0.7 and some above 0.7 , then some individuals must have observed information in favour of $H$ and the others information against $H$; so, intuitively, $\pi(H)$ should be higher than 0.7 if 'most' individuals had priors of $H$ below 0.7 (hence, had information supporting $H$ ).

These considerations highlight that knowing just the individuals' current (i.e. posterior) opinions $\pi_{1}, \ldots, \pi_{n}$ does not suffice to determine a collective opinion $\pi$ that efficiently aggregates private information. But $\pi_{1}, \ldots, \pi_{n}$ are all that classic opinion pooling takes into account in calculating $\pi$. This suggests that one should depart from the classic framework. As the above example lets one suspect, the collective opinion $\pi$ should be sensitive not just to people's posterior opinions $\pi_{1}, \ldots, \pi_{n}$ but also their prior opinions.

This paper (which is based on my unpublished paper Dietrich $2004^{1}$ ) presents an axiomatic framework that explicitly models the information states of the individuals. The axioms lead (in the common prior case) to a unique formula for the collective probability function; no weights or other parameters are needed to incorporate all individual information into the collective beliefs. For the reason explained above, the collective beliefs depend not just on people's actual (i.e. posterior) beliefs but also their prior beliefs. This increased individual input is necessary and sufficient to efficiently aggregate information, which might come as a surprise. In short, knowing the (complex) content of people's private information is not needed: knowing people's prior-posterior pairs suffices.

As an alternative to our approach, the supra-Bayesian approach might also be able to aggregate information efficiently; however, despite conceptual elegance, the approach suffers from some problems, amongst which practical infeasibility. ${ }^{2}$

In modelling both individuals and the collective as Bayesian rationals, our findings are also relevant to the theory of Bayesian aggregation, which aims to merge individual beliefs/values/preferences satisfying Bayesian rationality conditions (in the sense of Savage 1954 or Jeffrey 1983) into equally rational collective ones; for the ex ante approach, e.g. Seidenfeld et al. (1989), Broome (1990), Schervish et al. (1991) and Mongin (1995, 1998); for the ex post approach, e.g. Hylland and Zeckhauser (1979), Levi (1990), Hild (1998) and Risse (2001); for an excellent overview, see Risse (2003).

\footnotetext{
1 See also Marcus Pivato's (2008) related work mentioned in the acknowledgements.

2 In the supra-Bayesian approach (introduced by Morris' 1974 seminal work and extended in a large literature), collective beliefs are obtained as posterior probabilities (held by the real or virtual 'supra-Bayesian') conditional on the observed individual beliefs (treated as random events or evidence). This presupposes knowing (i) prior probabilities, and (ii) the likelihoods with which the individuals make probability assignments. It is not clear where these prior probabilities and likelihoods can come from; reaching a compromise or consensus on them might involve a more complex opinion pooling problem than the original one.
} 
Section 2 presents the axiomatic model and derives the resulting aggregation rule. Section 3 gives a numerical example. Section 4 identifies our pooling formula as a form of multiplicative opinion pooling. Sections 5 and 6 address the case of no common prior. Section 7 analyses the independent-information assumption made so far. Section 8 generalises the aggregation rule to arbitrary information overlaps.

\section{An axiomatic model}

Consider a group of persons $i=1, \ldots, n(n \geq 2)$ who need collective beliefs on certain hypotheses, represented as subsets $H$ of a non-empty set $\Omega$ of possible worlds, i.e. worlds that are possible under the shared information. Throughout I call information (knowledge, an observation etc.) 'shared' if it is held by all group members. Let $\mathcal{H}$ be the set of hypotheses $H \subseteq \Omega$ of interest, where $\mathcal{H}$ forms a finite or countably infinite partition of $\Omega$ and $\varnothing \notin \mathcal{H}$. So, the hypotheses are mutually exclusive and exhaustive. A simple but frequent case is a binary problem $\mathcal{H}=\{H, \Omega \backslash H\}$, where $H$ might be the hypothesis that the defendant in a court trial is guilty. In a non-binary case, $\mathcal{H}$ might contain different hypotheses on the defendant's extent of guilt.

In practice, the hypotheses on which opinions are formed need not be represented as subsets of a set of worlds $\Omega$. This representation and indeed the set $\Omega$ are needed only in the present formal framework, so that we can formulate axioms, and introduce further background objects (events and probability measures) which are needed in the axioms but do not appear when applying the resulting pooling formulas.

I call an opinion $($ on $\mathcal{H})$ any function $f: \mathcal{H} \rightarrow(0,1]$ with $\sum_{H \in \mathcal{H}} f(H)=1$ (whereas probability measures are, as usual, defined on $\sigma$-algebras of events ${ }^{3}$ ); let $\Pi$ be the set of all these functions $f$.

Let each individual $i$ hold an opinion $\pi_{i} \in \Pi$, and let the collective also hold an opinion $\pi \in \Pi$. So far, this is entirely classical. Classical opinion pooling would proceed by placing conditions on how $\pi$ depends on $\pi_{1}, \ldots, \pi_{n}$, resulting in a unique relationship (e.g. $\pi=\frac{1}{n} \pi_{1}+\cdots+\frac{1}{n} \pi_{n}$ ) or a class of possible relationships (e.g. all linear relationships).

\subsection{Simple case: common prior beliefs and a common belief domain}

Before stating the axiomatic approach in full generality (that is, before allowing individuals to hold different prior beliefs defined within different domains of events), I sketch the approach in the simple base-line case. Suppose for the moment that any individual $i$ 's opinion $\pi_{i}: \mathcal{H} \rightarrow(0,1]$ is given by

$$
\pi_{i}(H)=P\left(H \mid E_{i}\right) \quad \text { for all } H \in \mathcal{H},
$$

\footnotetext{
3 Any opinion uniquely extends to a probability measure defined on the $\sigma$-algebra $\sigma(\mathcal{H})$ generated by $\mathcal{H}$, and so we lose nothing by pooling opinions defined on $\mathcal{H}$ rather than probability measures defined on $\sigma(\mathcal{H})$. By definition, opinions never assign zero probability to any hypothesis; this is mainly for technical convenience.
} 
where for now $P$ is a common prior probability measure (defined on an appropriate $\sigma$-algebra over $\Omega$, for instance the power set $\mathcal{P}(\Omega)$ ), and where $E_{i} \subseteq \Omega$ is individual $i$ 's private information with $P\left(E_{i}\right)>0$. Suppose further that people hold independent information: $E_{1}, \ldots, E_{n}$ are independent conditional on any hypothesis $H \in \mathcal{H} .^{4}$ We would like to calculate a group opinion $\pi$. This group opinion should include all information spread over the individuals, i.e.

$$
\pi(H)=P\left(H \mid E_{1} \cap \ldots \cap E_{n}\right) \quad \text { for all } H \in \mathcal{H}
$$

(where one easily checks that (1) is well-defined, i.e. that $P\left(E_{1} \cap \ldots \cap E_{n}\right)>0$ ). One approach would be to ask all individuals $i$ to 'tell' their private experience $E_{i}$, so that the group could simply gather all experiences and calculate the conditional probabilities (1). But this procedure may be unrealistic, as personal experience may be very complex and hard-to-communicate in normal language and limited time. (Another problem, which we currently assume away by using a common belief domain, is that person $i$ 's experience $E_{i}$ may be an event of which the other persons have no prior beliefs, or even no awareness or conceptualisation; asymmetries in awareness or conceptualisation might indeed explain why different people make different experiences.)

Assuming that private evidence cannot (or is not) communicated, can the beliefs in (1) be calculated at all? The following derivation gives a positive answer. Consider a hypothesis $H \in \mathcal{H}$ and the belief $\pi(H)$ as defined by (1). Applying Bayes' rule and then our independence assumption,

$$
\begin{aligned}
\pi(H) & =\frac{P(H) P\left(E_{1} \cap \ldots \cap E_{n} \mid H\right)}{\sum_{H^{\prime} \in \mathcal{H}} P\left(H^{\prime}\right) P\left(E_{1} \cap \ldots \cap E_{n} \mid H^{\prime}\right)} \\
& =\frac{P(H) P\left(E_{1} \mid H\right) \cdots P\left(E_{n} \mid H\right)}{\sum_{H^{\prime} \in \mathcal{H}} P\left(H^{\prime}\right) P\left(E_{1} \mid H^{\prime}\right) \cdots P\left(E_{n} \mid H^{\prime}\right)} .
\end{aligned}
$$

In the numerator and the denominator, each factor of type $P\left(E_{i} \mid H\right)$ can be rewritten according to

$$
P\left(E_{i} \mid H\right)=\frac{P\left(H \mid E_{i}\right) P\left(E_{i}\right)}{P(H)}=\frac{\pi_{i}(H) P\left(E_{i}\right)}{P(H)} .
$$

\footnotetext{
4 Why do I assume that information is independent conditional on any hypothesis rather than unconditionally? Unconditional independence would be implausible. Suppose for instance that the information of individuals 1 and 2 both strongly correlate with the same hypothesis $H$ in $\mathcal{H}$. (In a jury trial, the jurors 1 and 2 might each observe patterns in the defendant's behaviour which strongly point towards the hypothesis of guilt.) Then $E_{1}$ and $E_{2}$ are usually not independent but positively correlated $\left(P\left(E_{2} \mid E_{1}\right)>P\left(E_{2}\right)\right)$, because learning $E_{1}$ raises the probability of $H$, which in turn raises that of $E_{2}$. More generally, since the evidences $E_{1}, \ldots, E_{n}$ tell something about the hypotheses, learning some of the $E_{i}$ s leads to revised probabilities of the hypotheses, which leads to revised probabilities of the other $E_{i} \mathrm{~s}$. In short, the $E_{i} \mathrm{~s}$ are non-independent because they are mutually relevant via their relevance to hypotheses in $\mathcal{H}$. This argument for non-independence is blocked once we condition on a hypothesis: conditional on a given hypothesis being true, evidences are not relevant to (i.e., do not bring new information about) hypotheses. If all existing probabilistic dependence between evidences goes 'via' the hypotheses, then conditioning on a hypothesis eliminates all sources of dependence, and the evidences become conditionally independent. Our (conditional) independence assumption is analysed again below.
} 
Substituting this expression, we obtain

$$
\begin{aligned}
\pi(H) & =\frac{P(H) \frac{\pi_{1}(H) P\left(E_{1}\right)}{P(H)} \cdots \frac{\pi_{n}(H) P\left(E_{n}\right)}{P(H)}}{\sum_{H^{\prime} \in \mathcal{H}} P\left(H^{\prime}\right) \frac{\pi_{1}\left(H^{\prime}\right) P\left(E_{1}\right)}{P\left(H^{\prime}\right)} \cdots \frac{\pi_{n}\left(H^{\prime}\right) P\left(E_{n}\right)}{P\left(H^{\prime}\right)}} \\
& =\frac{\pi_{1}(H) \cdots \pi_{n}(H) / P(H)^{n-1}}{\sum_{H^{\prime} \in \mathcal{H}} \pi_{1}\left(H^{\prime}\right) \cdots \pi_{n}\left(H^{\prime}\right) / P\left(H^{\prime}\right)^{n-1}} .
\end{aligned}
$$

Interestingly, any private information $E_{i}$ has dropped out altogether, so that the collective opinion $\pi$ can be calculated solely on the basis of the revealed individual opinions $\pi_{1}, \ldots, \pi_{n}$ (and the fixed prior). Put differently, each individual information $E_{i}$ has been incorporated without disclosing it. In short, denoting by $p$ the prior opinion $\left.P\right|_{\mathcal{H}}$ (i.e., the restriction of $P$ to the hypotheses of interest), we have shown that

$$
\pi \propto \pi_{1} \cdots \pi_{n} / p^{n-1} .
$$

Here and throughout, I call functions $f, g: \mathcal{H} \rightarrow \mathbb{R}$ proportional, written $f \propto g$, if there exists a constant $k \neq 0$ such that $f(H)=k g(H)$ for all $H \in \mathcal{H}$.

\subsection{General cse: possibly distinct prior beliefs and belief domains}

After this preliminary analysis, let us start afresh, this time in full generality, and stating all assumptions as explicit axioms. Recall that we consider individual opinions $\pi_{1}, \ldots, \pi_{n} \in \Pi$ and a collective opinion $\pi \in \Pi$. The further elements introduced in the preliminary Sect. 2.1 (namely, $P, E_{1}, \ldots, E_{n}$ ) are now re-introduced in their general and official form. For each person $i$ there is (without having to be revealed):

- $\quad$ an event $E_{i} \subseteq \Omega, i$ 's personal information;

- a ('prior') probability measure $P_{i}$ representing $i$ 's beliefs based on the shared information (hence prior to observing $E_{i}$ ). $P_{i}$ need not assign a probability to all events in $\mathcal{P}(\Omega)$; rather, $P_{i}$ is defined on some $\sigma$-algebra $\mathcal{A}_{i} \subseteq \mathcal{P}(\Omega)$, containing the events on which $i$ holds beliefs (whereas on other events $i$ may lack beliefs, or even lack awareness or conceptualisation). But $\mathcal{A}_{i}$ should contain at least $E_{i}$ and all hypotheses in $\mathcal{H}$, where $P_{i}\left(E_{i}\right)>0$ and $P_{i}(H)>0$ for all $H \in \mathcal{H}$. The restriction of $i$ 's prior belief $P_{i}$ to $\mathcal{H}$ is called $i$ 's prior opinion. It is denoted by $p_{i}(\in \Pi)$ and given by $p_{i}(H)=P_{i}(H)$ for all $H \in \mathcal{H}$.

These model resources allow us to state a standard rationality condition:

Individual Bayesian Rationality (IBR) $\pi_{i}(H)=P_{i}\left(H \mid E_{i}\right)$ for each person $i$ and hypothesis $H \in \mathcal{H}^{5}$

\footnotetext{
5 The conditional probability $P_{i}\left(H \mid E_{i}\right)$ is well-defined because $E_{i}, H \in \mathcal{A}_{i}$ and $P_{i}\left(E_{i}\right)>0$. Our assumptions also take care that all other conditional probabilities used in this paper are well-defined.
} 
A person $i$ 's belief domain $\mathcal{A}_{i}$ may fail to contain another person $j$ 's observation $E_{j}$, and this for (at least) two reasons. First, the fact that $j$ but not $i$ observed $E_{j}$ may be due precisely to $j$ having subjectively conceptualised $E_{j}$ but $i$ not having done so; juror $j$ in a trial may be the only juror to observe the suspicious smile on the defendant's face because the other jurors $i$ do not even know what a suspicious smile would be like. Second, $j$ 's information $E_{j}$ may be so detailed and complex that prior to $j$ observing it, it belonged not even to $j$ 's own belief domain, let alone to $i$ 's; that is, it was only while observing $E_{j}$ that person $j$ extended his prior beliefs to a larger domain $\mathcal{A}_{j}$ containing $E_{j}$.

The collective agent could be either a real agent (e.g., a social planner) who has to update his beliefs after learning the beliefs of the group members; this turns the model into a belief revision model. But the paper mainly interprets the collective agent as a virtual agent with its own beliefs. The social choice paradigm requires it to be as rational as any real individual. ' ${ }^{6}$ ationality' refers to different things in different contexts (e.g. to transitivity of preferences in Arrovian preference aggregation, to von-NeumannMorgenstern rationality in Harsanyi's Theorem on group preferences over lotteries, to logical consistency in judgment aggregation, and so on). In the present context, it naturally refers to Bayesian rationality. To formulate this, I suppose that there is

- a ('prior') probability measure $P$, representing collective beliefs based on people's shared information (hence not on their private information $E_{1}, \ldots, E_{n}$ ).P is defined on some $\sigma$-algebra $\mathcal{A} \subseteq \mathcal{P}(\Omega)$, the domain of the collective beliefs, which contains at least all private evidences $E_{1}, \ldots, E_{n}$ and all $H \in \mathcal{H}$, where $P\left(E_{1} \cap \ldots \cap E_{n}\right)>0$ and $P(H)>0$ for all $H \in \mathcal{H}$. The restriction of the collective prior belief $P$ to $\mathcal{H}$ is called the collective prior opinion; it is denoted by $p(\in \Pi)$ and given by $p(H)=P(H)$ for all $H \in \mathcal{H}$.

$\mathcal{A}, P$ and $p$ are the collective counterparts of $\mathcal{A}_{i}, P_{i}$ and $p_{i}$. The collective counterpart of IBR is:

Collective Bayesian Rationality (CBR) $\pi(H)=P\left(H \mid E_{1} \cap \ldots \cap E_{n}\right)$ for each hypothesis $H \in \mathcal{H}$.

Condition (CBR) requires the collective opinion $\pi$ to incorporate all information spread over people: the shared information (contained in the prior $P$ ) and all personal information (contained in $E_{1}, \ldots, E_{n}$ ).

While we have ensured, via (CBR), that the collective opinion uses all evidence scattered across individuals, we have done nothing so far to constrain the collective prior probability measure $P$ (which underlies $\pi$ ). Indeed, $P$ may so far be totally disconnected from the individual prior probability measures $P_{1}, \ldots, P_{n}$ (which underlie $\left.\pi_{1}, \ldots, \pi_{n}\right)$. The next condition does something to connect $P$ to $P_{1}, \ldots, P_{n}$. More precisely, the condition ties the likelihood that the collective assigns to the various individual evidences $E_{1}, \ldots, E_{n}$ to the individuals' own likelihood assessments:

\footnotetext{
6 The collective agent should be rational notably because it forms the basis for collective actions and decisions.
} 
Accept People's Likelihood Assessments (APLA) For all persons $i$ and hypotheses $H \in \mathcal{H}, P\left(E_{i} \mid H\right)=P_{i}\left(E_{i} \mid H\right)$.

This principle requires the collective to take over $i$ 's own interpretation of $i$ 's information $E_{i}$ as given by $i$ 's likelihood assignments $P_{i}\left(E_{i} \mid H\right), H \in \mathcal{H}$. To motivate this condition, let me first explain the context in a little more detail. In statistics, the information that data contain on given hypotheses (as opposed to prior beliefs on these hypotheses) is usually taken to be summarised in the data's likelihood function, which maps any hypothesis to the data's probability given this hypothesis. For instance, the information on humidity contained in a temperature measurement of $20^{\circ} \mathrm{C}$ is given by the mapping that assigns to each potential humidity level the probability that temperature is $20^{\circ} \mathrm{C}$ given this humidity level. In our case, the information contained in individual $i$ 's evidence $E_{i}$ is summarised in $E_{i}$ 's likelihood function, mapping any hypothesis $H$ to $E_{i}$ 's probability given $H$. But how large exactly is $E_{i}$ 's probability given $H$ ? For instance, how probable is it that the defendant in a trial has a particular facial expression $\left(E_{i}\right)$ given the hypothesis that he is guilty $(H)$ ? The answer may be far from trivial, as one might come up with various different interpretations of the same observation. Condition (APLA) requires that the answer that the collective gives matches the answer that the individual who observed the evidence gives; that is, $P\left(E_{i} \mid H\right)=P_{i}\left(E_{i} \mid H\right)$. What is the motivation behind identifying $P\left(E_{i} \mid H\right)$ with $P_{i}\left(E_{i} \mid H\right)$ ? Why not also take other persons' interpretations of $E_{i}$ into account by defining $P\left(E_{i} \mid H\right)$ as some compromise of $P_{1}\left(E_{i} \mid H\right), \ldots, P_{n}\left(E_{i} \mid H\right)$ ? First, for reasons explained above, the persons $j \neq i$ may not even hold beliefs on the unobserved event $E_{i}$ (i.e., $\left.E_{i} \notin \mathcal{A}_{j}\right)$, in which case $P_{j}\left(E_{i} \mid H\right)$ is simply undefined. Second, assuming that the persons $j \neq i$ do hold such beliefs (i.e., $E_{i} \in \mathcal{A}_{j}$ ), a 'likelihood compromise' could only be formed after each person $j$ reveals $P_{j}\left(E_{i} \mid H\right)$; which in turn supposes that first $i$ communicates his informational basis $E_{i}$ in all detail to the rest of the group. This is not only at odds with the present approach, but may also be infeasible: given the possible complexity of $E_{i}$ and the limitations of language, time, $i$ 's ability to describe $E_{i}, j$ 's $(j \neq i)$ ability to understand $E_{i}$ and so on, $j$ could probably learn at most some approximation $\tilde{E}_{i}$ of $E_{i}$, and so $j$ could at most provide $j$ 's likelihood of $\tilde{E}_{i}$, which only approximates $j$ 's likelihood of the true $E_{i}\left(P_{j}\left(\tilde{E}_{i} \mid H\right) \approx P_{j}\left(E_{i} \mid H\right)\right)$.

The next assumption is not a normative condition but rather an assumption on the environment: individuals receive independent information. This assumption will be analysed (and relaxed) in later sections; see footnote 4 above for first considerations. For now, I only mention that it is strong but very common. It is analogous to (i) independence assumptions on private information/types in Bayesian games, (ii) the independence condition in the literature on the Condorcet Jury Theorem (see Dietrich 2008 for a critique of the condition), (iii) the Parental Markov Condition in the theory of Bayesian networks (interpreting the true hypothesis in $\mathcal{H}$ as the parent of each information $E_{i}$ in a Bayesian network; see Pearl 2000) and (iv) Fitelson'sFitelson (2001) condition of confirmational independence. 
Independent Information (Ind) For each hypothesis $H \in \mathcal{H}$, the personal observations $E_{1}, \ldots, E_{n}$ are independent conditional on $H .^{7}$

I am ready to state the theorem. Recall that $p_{i}, \pi_{i}$ is the pair of person $i$ 's prior and posterior opinion, and $p, \pi$ is the pair of the collective prior and posterior opinion.

Theorem 1 Suppose individuals satisfy (IBR), information satisfies (Ind), and the collective satisfies (CBR) and (APLA). Then the collective opinion $\pi$ is proportional to the collective prior opinion times all individual posterior-to-prior ratios:

$$
\pi \propto p \frac{\pi_{1}}{p_{1}} \cdots \frac{\pi_{n}}{p_{n}} .
$$

Proof Suppose (IBR), (CBR), (APLA) and (Ind) hold. For all $H$ in $\mathcal{H}$,

$$
\begin{aligned}
\pi(H) & =P\left(H \mid E_{1} \cap \cdots \cap E_{n}\right) \text { by }(\mathrm{CBR}) \\
& =\frac{P\left(E_{1} \cap \cdots \cap E_{n} \mid H\right) p(H)}{P\left(E_{1} \cap \cdots \cap E_{n}\right)} \text { by Bayes' rule } \\
& =k P\left(E_{1} \cap \cdots \cap E_{n} \mid H\right) p(H) \text { for a constant } k \neq 0 \\
& =k P\left(E_{1} \mid H\right) \cdots P\left(E_{n} \mid H\right) p(H) \text { by (Ind) } \\
& =k P_{1}\left(E_{1} \mid H\right) \cdots P_{n}\left(E_{n} \mid H\right) p(H) \text { by (APLA) } \\
& =k \frac{P_{1}\left(H \mid E_{1}\right) P_{1}\left(E_{1}\right)}{p_{1}(H)} \cdots \frac{P_{n}\left(H \mid E_{n}\right) P_{n}\left(E_{n}\right)}{p_{n}(H)} p(H) \text { by Bayes' rule } \\
& =k^{\prime} \frac{P_{1}\left(H \mid E_{1}\right)}{p_{1}(H)} \cdots \frac{P_{n}\left(H \mid E_{n}\right)}{p_{n}(H)} p(H) \text { for a constant } k^{\prime} \neq 0 \\
& =k^{\prime} \frac{\pi_{1}(H)}{p_{1}(H)} \cdots \frac{\pi_{n}(H)}{p_{n}(H)} p(H) \text { by (IBR). }
\end{aligned}
$$

Three important remarks are due.

1. As promised, the collective opinion $\pi$ is calculated without people having to communicate their arbitrarily complex informational bases $E_{i}$ or their likelihoods $P\left(E_{i} \mid H\right), H \in \mathcal{H}$. In practice, all persons $i$ submit their prior-posterior pairs $p_{i}, \pi_{i}$ (or just their ratios $\pi_{i} / p_{i}$ ), and then the collective opinion $\pi$ is calculated. So, compared to standard opinion pooling, we additionally require submission of prior opinions $p_{1}, \ldots, p_{n}$, a complication that enables the incorporation of the individual information $E_{1}, \ldots, E_{n}$ into the collective opinion.

2. Theorem 1's formula does not fully solve the aggregation problem since we do not yet know how to determine the collective prior opinion $p$. Strategies to choose $p$ are presented in Sects. 5 and 6. In practice, there is an alternative to having to choose $p$ : one might use an approximation of Theorem 1's formula, defining the collective opinion as

\footnotetext{
7 As usual, by 'independence’ of events I mean full independence, not just pairwise independence.
} 


$$
\pi_{\text {approx }} \propto \frac{\pi_{1}}{p_{1}} \cdots \frac{\pi_{n}}{p_{n}}
$$

the product of all posterior-to-prior ratios. When and why can $\pi_{\text {approx }}$ count as a good approximation of Theorem 1's formula? Let me give some heuristic arguments. ${ }^{8}$ Often, the function $\frac{\pi_{1}}{p_{1}} \cdots \frac{\pi_{n}}{p_{n}}$ varies considerably, i.e. assigns very different values to the hypotheses $H$ in $\mathcal{H}$. Intuitively, this is because pooled information is often strong evidence for or against certain hypotheses. More formally, if sufficiently many of the individual ratios $\frac{\pi_{i}}{p_{i}}$ vary at least moderately, the product $\frac{\pi_{1}}{p_{1}} \cdots \frac{\pi_{n}}{p_{n}}$ varies strongly (provided that the individual variations do not systematically cancel each other out). ${ }^{9}$ Whenever the variation of $\frac{\pi_{1}}{p_{1}} \cdots \frac{\pi_{n}}{p_{n}}$ is strong enough to 'outweigh' that of $p$ (assuming $p$ should not vary very much), the function $\frac{\pi_{1}}{p_{1}} \cdots \frac{\pi_{n}}{p_{n}}$ varies roughly like $p \frac{\pi_{1}}{p_{1}} \cdots \frac{\pi_{n}}{p_{n}}$; and hence, the opinions $\pi_{\text {approx }}$ and $\pi$ (obtained by normalising the two functions so as to each sum to one) are roughly similar.

3. Assume a unanimous posterior agreement $\pi_{1}=\cdots=\pi_{n}$ (as in the introduction's example). Then only in special cases does $\pi$ equal $\pi_{1}=\cdots=\pi_{n}$, which shows that the unanimity/Pareto principle often required in standard opinion pooling is problematic under informational asymmetries. One such special case is that $\pi_{1}=\cdots=\pi_{n}=p_{1}=\cdots=p_{n}=p$, so that none of the personal observations $E_{1}, \ldots, E_{n}$ confirms or disconfirms any hypothesis, i.e. in essence, there is no informational asymmetry.

An important special case of Theorem 1 is that where people have managed to agree on how to interpret their shared information, i.e. where they hold a common prior opinion:

Common Prior (CP) $p_{1}=\cdots=p_{n}=p$ (i.e., the prior probability measures $P_{1}, \ldots, P_{n}, P$ agree on all hypotheses in $\mathcal{H}$, though perhaps not elsewhere).

Corollary 1 Under the assumptions of Theorem 1 and (CP), the collective opinion $\pi$ is given by

$$
\pi \propto \pi_{1} \cdots \pi_{n} / p_{1}^{n-1}
$$

Let me make three remarks on this corollary.

1. The corollary's formula differs in an important respect from Theorem 1's formula: the parameter $p$ has been eliminated, and so the collective opinion $\pi$ is fully determined by the individual prior and posterior opinions. By contrast, if (CP) fails, i.e. if the group did not manage to agree on how to interpret the shared

\footnotetext{
8 I owe these thoughts to the helpful referee.

9 If for instance, most individual ratios peak at the same hypothesis (say, if most jurors believe the defendant is guilty) then the product of ratios is likely to strongly peak at this hypothesis.
} 
information, Theorem 1's formula does not fully solve the aggregation problem, as we need a way to determine the collective prior $p$ (see Sects. 5 and 6).

2. Condition $(\mathrm{CP})$ can in fact be seen as the conjunction of two conditions. The first (descriptive) condition is that $p_{1}=\cdots=p_{n}$, i.e. all persons $i$ submit the same prior opinion. The second (normative) condition is that the unanimity (or Pareto) principle holds for the prior opinions, i.e. if all submit the same prior opinion, this becomes the collective prior opinion. Applying a unanimity condition to prior opinions is far less problematic than doing so for the posterior opinions $\pi_{1}, \ldots, \pi_{n}, \pi$, because prior opinions contain no informational asymmetry.

3. According to a prominent view, held notably by Harsanyi, any inter-personal differences in beliefs between rational agents stem from different information (in a suitably general sense of this word), never from different prior beliefs. ${ }^{10}$ If this view is correct, and if each opinion $p_{i}$ indeed incorporates no information except the shared one which does not depend on $i$, then the $p_{i}$ s must be identical. So, Harsanyi's view places us in the comfortable position of being able to assume (CP). Harsanyi's view is based on modelling all experiences-including early ones in life and perhaps prenatal ones-as information shaping beliefs via Bayesian conditioning. If we think of $i$ 's private information $E_{i}$ as containing all such experiences, then the events $E_{i}$ (and the underlying space $\Omega$ ) inevitably become highly complex. While this by itself poses no problem (since $E_{1}, \ldots, E_{n}$ need not be revealed), at least one difficulty arises. Even if Harsanyi were fundamentally right, people will in practice often not agree on a common prior opinion, if only because they do not 'remember' the common prior opinion that they used to hold at the prenatal stage. I personally do not share Harsanyi's view. I believe in the possibility of genuinely non-information-driven disagreements, and hence in the possibility that $p_{1}, \ldots, p_{n}$ differ in spite of incorporating the same (shared) information. $^{11}$

4. Instead of interpreting $E_{i}$ as reflecting all of $i$ 's personal information, one might re-interpret $E_{i}$ as reflecting only that part of $i$ 's personal information which $i$ has incorporated rationally into his opinion (in the sense of Bayesian conditioning). Then IBR becomes true by definition. All not rationally incorporated personal

\footnotetext{
$10 \mathrm{I}$ am grateful to the referee for raising this issue.

11 Harsanyi's claim is true nearly by definition if the notion of 'information' is purely technical and if the claim is taken to be one about mathematically representing different probability measures as being obtained by conditioning from a common probability measure (defined on a suitably extended algebra of events). Under a so-extended notion of 'information', the whole process of personal deliberation needed to form one's beliefs and to interpret one's information constitutes another large piece of ('meta-')information. The current paper's notion of 'information' is not of this abstract kind. It is a substantive notion, under which it is possible that two opinions incorporate no (or the same) information and yet differ. If, however, Harsanyi's claim is taken to be not just about mathematical representability but about the psychological reality of rational agents, then the claim is problematic. Many experiences in life (such as hearing a sound for the first time) have a content that was not previously conceptualised by the agent, hence cannot belong to the algebra within which the agent previously held beliefs. So the agent's new beliefs after the experience cannot stem from updating the old beliefs by Bayesian conditioning on this event. The topic of non-informational belief formation goes beyond this paper (but will be developed in the paper 'A reason-based theory of rational belief' with Christian List).
} 
Table 1 Collective probability $\pi^{H}=\pi(H)$ in dependence of the common prior $p^{H}=p(H)$ and the individual posteriors $\pi_{i}^{H}=\pi_{i}(H)$, for a group of size $n=2$

\begin{tabular}{llllll}
\hline$\pi_{1}^{H}, \pi_{2}^{H}$ & $\mathrm{p}^{H}$ & & & & \\
\cline { 2 - 6 } & 0.1 & 0.25 & 0.5 & 0.75 & 0.9 \\
\hline $0.1,0.1$ & 0.1 & 0.036 & 0.012 & 0.004 & 0.001 \\
$0.25,0.1$ & 0.25 & 0.1 & 0.036 & 0.012 & 0.004 \\
$0.25,0.25$ & 0.5 & 0.25 & 0.1 & 0.036 & 0.012 \\
$0.5,0.1$ & 0.5 & 0.25 & 0.1 & 0.036 & 0.012 \\
$0.5,0.25$ & 0.75 & 0.5 & 0.25 & 0.1 & 0.036 \\
$0.5,0.5$ & 0.9 & 0.75 & 0.5 & 0.25 & 0.1 \\
$0.75,0.1$ & 0.75 & 0.5 & 0.25 & 0.1 & 0.036 \\
$0.75,0.25$ & 0.9 & 0.75 & 0.5 & 0.25 & 0.1 \\
$0.75,0.5$ & 0.964 & 0.9 & 0.75 & 0.5 & 0.25 \\
$0.75,0.75$ & 0.988 & 0.964 & 0.9 & 0.75 & 0.5 \\
$0.9,0.1$ & 0.9 & 0.75 & 0.5 & 0.25 & 0.1 \\
$0.9,0.25$ & 0.964 & 0.9 & 0.75 & 0.5 & 0.25 \\
$0.9,0.5$ & 0.988 & 0.964 & 0.9 & 0.75 & 0.5 \\
$0.9,0.75$ & 0.996 & 0.988 & 0.964 & 0.9 & 0.75 \\
$0.9,0.9$ & 0.999 & 0.996 & 0.988 & 0.964 & 0.9 \\
\hline
\end{tabular}

information is then simply thrown away, i.e. not included in individual or collective beliefs. ${ }^{12}$

\section{A numerical example for a simple case}

Consider the simple case of a binary problem $\mathcal{H}=\{H, \Omega \backslash H\}$ ( $H$ and $\Omega \backslash H$ might mean that the defendant in a court trial is guilty, respectively, innocent, and persons might be jurors). Suppose Common Prior (CP), i.e. $p_{1}=\cdots=p_{n}=p$. By Theorem 1 (that is, by its corollary), the collective posterior of $H$ is given by

$$
\begin{aligned}
\pi^{H} & =\frac{\pi_{1}^{H} \cdots \pi_{n}^{H} /\left(p^{H}\right)^{n-1}}{\pi_{1}^{H} \cdots \pi_{n}^{H} /\left(p^{H}\right)^{n-1}+\left(1-\pi_{1}^{H}\right) \cdots\left(1-\pi_{n}^{H}\right) /\left(1-p^{H}\right)^{n-1}} \\
& =\frac{1}{1+\left(1 / \pi_{1}^{H}-1\right) \cdots\left(1 / \pi_{n}^{H}-1\right) /\left(1 / p^{H}-1\right)^{n-1}},
\end{aligned}
$$

where $p^{H}:=p(H), \pi^{H}:=\pi(H)$ and $\pi_{i}^{H}:=\pi_{i}(H)$.

\footnotetext{
12 Instead of throwing this information away, one might ask people to incorporate it (in some non-Bayesian ways, unfortunately) in the submitted prior opinions. This removes the informational symmetry underlying the submitted prior opinions, which in turn affects the interpretation and plausibility of the analysis of later sections.
} 
For the case of only $n=2$ individuals, in which the formula (3) for the collective posterior reduces to

$$
\begin{aligned}
\pi^{H} & =\frac{\pi_{1}^{H} \pi_{2}^{H} / p^{H}}{\pi_{1}^{H} \pi_{2}^{H} / p^{H}+\left(1-\pi_{1}^{H}\right)\left(1-\pi_{2}^{H}\right) /\left(1-p^{H}\right)} \\
& =\frac{1}{1+\left(1 / \pi_{1}^{H}-1\right)\left(1 / \pi_{2}^{H}-1\right) /\left(1 / p^{H}-1\right)},
\end{aligned}
$$

Table 1 contains the values of the collective belief $\pi^{H}$ for all possible combinations of values of $p^{H}, \pi_{1}^{H}, \pi_{2}^{H}$ in the grid $\{0.1,0.25,0.5,0.75,0.9\} .{ }^{13}$ Note how drastically the group belief $\pi^{H}$ depends on the prior $p^{H}$. By shifting $p^{H}$ below (above) the individual posteriors $\pi_{i}^{H}$, the group belief $\pi^{H}$ quickly approaches $1(0)$. The interpretation is that if the posteriors $\pi_{i}^{H}$ are all to the same side of the prior, then the evidences $E_{i}$ all point into the same direction, so that their conjunction points even more into that direction. But if the prior $p^{H}$ is somewhere in the middle of the posteriors $\pi_{i}^{H}$, the group belief $\pi^{H}$ may be moderate. The interpretation is that if some of the posteriors $\pi_{i}^{H}$ are above the prior and others are below the prior, then the evidences $E_{i}$ point into different directions, and their conjunction need not strongly point into any direction. The above formula for the group belief $\pi^{H}$ shows that it strictly increases as a function of each individual belief $\pi_{i}^{H}$, but strictly decreases as a function of the prior belief $p^{H}$. But how can one make sense of the group posterior $\pi^{H}$ depending negatively on the prior $p^{H}$ ? How could more prior support for $H$ possibly reduce $H$ 's posterior probability? The answer is that by increasing the prior $p^{H}$ while keeping the individual posteriors $\pi_{i}^{H}$ fixed one implicitly reduces the support that each of the evidences $E_{i}$ gives to $H$; as a result, the collective posterior of $H$ falls, intuitively because the reduced evidential support for $H$ overcompensates the increased prior support.

\section{Multiplicative opinion pooling}

If we treat the prior opinions $p_{1}, \ldots, p_{n}, p$ as fixed parameters, the pooling formula of Theorem 1 depends just on $\pi_{1}, \ldots, \pi_{n}$, hence defines a classic pooling function $F: \Pi^{n} \rightarrow \Pi$. Specifically, this pooling function is given by $\pi=g \cdot \pi_{1} \ldots \pi_{n}$ where $g$ is a fixed function on $\mathcal{H}$ given by $g \propto p /\left(p_{1} \cdots p_{n}\right)$ (and in particular as $g \propto p^{1-n}$ under Common Prior (CP)). So, our axioms lead to what one might call a multiplicative opinion pool. Formally, a (classic) opinion pool $F: \Pi^{n} \rightarrow \Pi$ is multiplicative if it is given by

$$
F\left(\pi_{1}, \ldots, \pi_{n}\right)=g \cdot \pi_{1} \cdots \pi_{n} \text { for all } \pi_{1}, \ldots, \pi_{n} \in \Pi,
$$

\footnotetext{
13 The entries are rounded results if three decimal digits are reported, and exact results else.
} 
for some fixed function $g: \mathcal{H} \rightarrow(0, \infty)$. The simplest multiplicative rule is that in which $g$ is a constant function, so that

$$
F\left(\pi_{1}, \ldots, \pi_{n}\right) \propto \pi_{1} \ldots \pi_{n} \text { for all } \pi_{1}, \cdots, \pi_{n} \in \Pi \text {. }
$$

Note how multiplicative opinion pools differ from the more common linear and geometric opinion pools; these arise from different axiomatic systems that do not make information explicit.

In fact, our axioms not only imply that pooling is multiplicative: they characterise multiplicative pooling if $\mathcal{H}$ is finite because every multiplicative rule can be obtained from suitable priors $p_{1}, \ldots, p_{n}, p \in \Pi .^{14}$

\section{Choosing the collective prior $p$ when there is no common prior}

If the interpretation of the shared information is controversial and hence $(\mathrm{CP})$ fails, the group needs to determine the collective prior $p$ in Theorem 1's formula. At least three strategies are imaginable. First, one might define $p$ as a uniform or maximum-entropy prior if available. Second, someone, not necessarily a group member, may be appointed to choose $p$, either by drawing on his own prior beliefs, or by taking inspiration from the submitted priors $p_{1}, \ldots, p_{n}$, or by using statistical estimation techniques if available. These two solutions have obvious limitations, including some ad-hoc-ness and a lack of democracy. A third alternative is to replace $p$ by $F\left(p_{1}, \ldots, p_{n}\right)$ and thus define the collective opinion by

$$
\pi \propto \frac{\pi_{1}}{p_{1}} \cdots \frac{\pi_{n}}{p_{n}} F\left(p_{1}, \ldots, p_{n}\right),
$$

where $F: \Pi^{n} \rightarrow \Pi$ is a standard opinion pool. Note that $F$ is used here not to aggregate people's actual (posterior) opinions $\pi_{1}, \ldots, \pi_{n}$ but to aggregate their prior opinions $p_{1}, \ldots, p_{n}$, namely into a 'compromise prior'. At first sight, one may wonder what is gained by formula (4) compared to the standard approach of defining $\pi=F\left(\pi_{1}, \ldots, \pi_{n}\right)$ without having to care about priors $p_{1}, \ldots, p_{n}$. Does formula (4) not just shift the classic aggregation problem-pooling $\pi_{1}, \ldots, \pi_{n}$ into $\pi$ - towards an equally complex aggregation problem about priors - pooling $p_{1}, \ldots, p_{n}$ into $p$ ? In an important respect, pooling $p_{1}, \ldots, p_{n}$ is simpler than pooling $\pi_{1}, \ldots, \pi_{n}$ : unlike $\pi_{1}, \ldots, \pi_{n}$, the prior opinions $p_{1}, \ldots, p_{n}$ involve no informational asymmetry since each $p_{i}$ is based on the same (shared) information. ${ }^{15}$ Hence any disagreement between $p_{1}, \ldots, p_{n}$ is due solely to different interpretations of that same body of information. This may facilitate the choice of $F$. For instance, aggregation may be guided by the

\footnotetext{
14 For any multiplicative rule $F: \Pi^{n} \rightarrow \Pi$, say generated by the function $g$, if we (for instance) take $p_{1}, \ldots, p_{n}, p$ to be all identical and proportional to $g^{-1 /(n-1)}$, then $g \propto p /\left(p_{1} \ldots p_{n}\right)$, and hence the multiplicative rule generated by $g$ coincides with that arising in Theorem 1.

15 One might even argue that while pooling $p_{1}, \ldots, p_{n}$ into $p$ is possible without using extra information (due to the informational symmetry), pooling $\pi_{1}, \ldots, \pi_{n}$ into $\pi$ is impossible without extra information (such as $p_{1}, \ldots, p_{n}$ ).
} 
unanimity/Pareto principle (which is problematic under informational asymmetry, as we have seen). Further, aggregation may place equal weights on each of the priors $p_{1}, \ldots, p_{n}$ (whereas pooling $\pi_{1}, \ldots, \pi_{n}$ may involve the difficult and vague exercise of assigning more weight to better informed people). The literature's two most prominent types of opinion pools $F: \Pi^{n} \rightarrow \Pi$ are

$$
\begin{array}{ll}
\text { linear opinion pools: } & F\left(p_{1}, \ldots, p_{n}\right)=w_{1} p_{1}+\ldots+w_{n} p_{n}, \\
\text { geometric opinion pools: } & F\left(p_{1}, \ldots, p_{n}\right) \propto p_{1}^{w_{1}} \ldots p_{n}^{w_{n}},
\end{array}
$$

with weights $w_{1}, \ldots, w_{i} \in[0,1]$ that add up to 1 (where in the geometric pool the factor of proportionality is chosen such that $\left.\sum_{H \in \mathcal{H}} F\left(p_{1}, \ldots, p_{n}\right)(H)=1\right)$. If $F$ is a linear, respectively, geometric opinion pool, our pooling formula (4) becomes

$$
\begin{aligned}
\pi & =\frac{\pi_{1}}{p_{1}} \cdots \frac{\pi_{n}}{p_{n}}\left(w_{1} p_{1}+\cdots+w_{n} p_{n}\right) \\
\text { resp. } \pi & \propto \frac{\pi_{1}}{p_{1}} \cdots \frac{\pi_{n}}{p_{n}} p_{1}^{w_{1}} \cdots p_{n}^{w_{n}}=\frac{\pi_{1}}{p_{1}^{1-w_{1}}} \cdots \frac{\pi_{n}}{p_{n}^{1-w_{n}}} .
\end{aligned}
$$

How should the weights $w_{1}, \ldots, w_{n}$ be chosen in practice? In general, unequal weights may be justified either by different information states or by different competence, i.e. ability to interpret information. The former reason does not apply here, since $p_{1}, \ldots, p_{n}$ are by definition based on the same (shared) information. If, in addition, differences of competence are either inexistent, or unknown, or not to be taken into account for reasons of procedural fairness, then equal weights $w_{1}=\cdots=w_{n}=1 / n$ are justified, so that our pooling formula becomes

$$
\begin{aligned}
\pi & =\frac{1}{n} \frac{\pi_{1}}{p_{1}} \cdots \frac{\pi_{n}}{p_{n}}\left(p_{1}+\cdots+p_{n}\right) \\
\text { resp. } \pi & \propto \frac{\pi_{1}}{p_{1}^{1-1 / n}} \cdots \frac{\pi_{n}}{p_{n}^{1-1 / n}},
\end{aligned}
$$

which is parameter-free, hence uniquely solves the aggregation problem.

\section{External and internal Bayesianity}

I now give an argument in defence of defining $F$ in (4) as a geometric (or more generally, externally Bayesian) opinion pool, hence in defence of our pooling formulae (6) and (8). Note first that in (4) $\pi$ is a function of the vector $\left(p_{1}, \pi_{1} \ldots, p_{n}, \pi_{n}\right) \in$ $(\Pi \times \Pi)^{n}=\Pi^{2 n}$, containing every person's prior and posterior.

Definition 1 A generalised opinion pool ('GOP') or generalised probability aggregation rule is a function $G: \Pi^{2 n} \rightarrow \Pi$.

Unlike a standard opinion pool $F: \Pi^{n} \rightarrow \Pi$, a GOP $G$ also takes as inputs the $p_{i} \mathrm{~s}$, i.e. people's interpretations of the shared information. As shown above, our axioms 
imply that a GOP $G$ should take the form (4), i.e. the form

$$
G\left(p_{1}, \pi_{1}, \ldots, p_{n}, \pi_{n}\right) \propto \frac{\pi_{1}}{p_{1}} \cdots \frac{\pi_{n}}{p_{n}} F\left(p_{1}, \ldots, p_{n}\right)
$$

where $F: \Pi^{n} \rightarrow \Pi$ is a standard opinion pool that merges the priors $p_{1}, \ldots, p_{n}$.

From a Bayesian perspective, two natural conditions may be imposed on a GOP, to be called external and internal Bayesianity. The former is an analogue of the equally named classic condition for standard opinion pools $F$ : it should not matter whether information arrives before or after pooling, i.e. pooling should commute with Bayesian updating. Formally, for every opinion $p \in \Pi$ and (likelihood) function $l: \mathcal{H} \rightarrow(0,1]$ the (updated) opinion $p^{l} \in \Pi$ is defined by

$$
p^{l}(H):=\frac{l(H) p(H)}{\sum_{H^{\prime} \in \mathcal{H}} l\left(H^{\prime}\right) p\left(H^{\prime}\right)}, \quad \text { in short } p^{l} \propto l p .
$$

Here, $l$ is interpreted as a likelihood function $P(E \mid$.) for some observation $E$, so that $p^{l}$ is a posterior probability. A standard opinion pool $F: \Pi^{n} \rightarrow \Pi$ is called externally Bayesian if

$$
F\left(p_{1}^{l}, \ldots, p_{n}^{l}\right)=F\left(p_{1}, \ldots, p_{n}\right)^{l}
$$

for every profile $\left(p_{1}, \ldots, p_{n}\right) \in \Pi^{n}$ and (likelihood) function $l: \mathcal{H} \rightarrow(0,1]$ (Madansky 1964). In particular, geometric opinion pools are externally Bayesian. An analogous concept can be defined for GOPs:

Definition 2 A GOP $G: \Pi^{2 n} \rightarrow \Pi$ is called externally Bayesian if

$$
G\left(p_{1}^{l}, \pi_{1}^{l}, \ldots, p_{n}^{l}, \pi_{n}^{l}\right)=G\left(p_{1}, \pi_{1}, \ldots, p_{n}, \pi_{n}\right)^{l}
$$

for every profile $\left(p_{1}, \pi_{1}, \ldots, p_{n}, \pi_{n}\right) \in \Pi^{2 n}$ and (likelihood) function $l: \mathcal{H} \rightarrow(0,1]$.

On the left hand side of this equation, not only all posteriors are updated $\left(\pi_{i}^{l}\right)$ but also all priors $\left(p_{i}^{l}\right)$, because the incoming information is observed by everybody, hence part of the shared information, hence contained in the priors.

While external Bayesianity requires that it be irrelevant whether pooling happens before or after updating, a different question is whether it matters who in the group has observed a given information. Internal Bayesianity requires that it be irrelevant whether every or just a single person obtains a given information:

Definition 3 A GOP $G: \Pi^{2 n} \rightarrow \Pi$ is called internally Bayesian if, for each person $i$

$G\left(p_{1}, \pi_{1}, \ldots, p_{i-1}, \pi_{i-1}, p_{i}, \pi_{i}^{l}, p_{i+1}, \pi_{i+1}, \ldots, p_{n}, \pi_{n}\right)=G\left(p_{1}^{l}, \pi_{1}^{l}, \ldots, p_{n}^{l}, \pi_{n}^{l}\right)$

for every profile $\left(p_{1}, \pi_{1}, \ldots, p_{n}, \pi_{n}\right) \in \Pi^{2 n}$ and (likelihood) function $l: \mathcal{H} \rightarrow(0,1]$. 
On the left hand side of this equation, $i$ 's prior is not updated $\left(p_{i}\right.$, not $\left.p_{i}^{l}\right)$, because the incoming information, being observed just by person $i$, is not part of the shared information, hence not reflected in any prior. Internal Bayesianity is based on the idea that the collective probabilities should incorporate all information available somewhere in the group, whether it is held by a single or every person. External and internal Bayesianity together imply that, for each person $i$,

$G\left(p_{1}, \pi_{1}, \ldots, p_{i-1}, \pi_{i-1}, p_{i}, \pi_{i}^{l}, p_{i+1}, \pi_{i+1}, \ldots, p_{n}, \pi_{n}\right)=G\left(p_{1}, \pi_{1}, \ldots, p_{n}, \pi_{n}\right)^{l}$

for every profile $\left(p_{1}, \pi_{1}, \ldots, p_{n}, \pi_{n}\right) \in \Pi^{2 n}$ and (likelihood) function $l: \mathcal{H} \rightarrow(0,1]$.

It turns out that if a GOP $G$ takes the form (9), then external and internal Bayesianity are in fact equivalent, and equivalent to external Bayesianity of $F$ :

Theorem 2 If a generalised opinion pool $G: \Pi^{2 n} \rightarrow \Pi$ has the form (9) where $F: \Pi^{n} \rightarrow \Pi$ is any opinion pool, the following conditions are equivalent:

(i) $G$ is externally Bayesian;

(ii) $G$ is internally Bayesian;

(iii) $F$ is externally Bayesian.

So, if one desires $G$ to be externally or internally Bayesian, one is bound to use an externally Bayesian opinion pool $F$ in our pooling formula (9), for instance a geometric opinion pool $F$, which leads to pooling formula (6), hence to (8) in the equal-weight case. There also exist more complex (non-geometric) externally Bayesian opinion pools $F$, characterised in full generality by Genest et al. (1986, Theorem 2.5), but geometric ones become the only solutions if $|\mathcal{H}| \geq 3$ and $F$ has some additional properties (see Genest et al. 1986, Corollary 4.5).

Proof I show that (i) is equivalent with each of (ii) and (iii). By (9),

$$
G\left(p_{1}^{l}, \pi_{1}^{l}, \ldots, p_{n}^{l}, \pi_{n}^{l}\right) \propto \frac{\pi_{1}^{l}}{p_{1}^{l}} \cdots \frac{\pi_{n}^{l}}{p_{n}^{l}} F\left(p_{1}^{l}, \ldots, p_{n}^{l}\right)
$$

and hence by (10)

$$
G\left(p_{1}^{l}, \pi_{1}^{l}, \ldots, p_{n}^{l}, \pi_{n}^{l}\right) \propto \frac{l \pi_{1}}{l p_{1}} \ldots \frac{l \pi_{n}}{l p_{n}} F\left(p_{1}^{l}, \ldots, p_{n}^{l}\right)=\frac{\pi_{1}}{p_{1}} \ldots \frac{\pi_{n}}{p_{n}} F\left(p_{1}^{l}, \ldots, p_{n}^{l}\right) .
$$

On the other hand, again by (9) and (10),

$$
G\left(p_{1}, \pi_{1}, \ldots, p_{n}, \pi_{n}\right)^{l} \propto l \frac{\pi_{1}}{p_{1}} \ldots \frac{\pi_{n}}{p_{n}} F\left(p_{1}, \ldots, p_{n}\right) \propto \frac{\pi_{1}}{p_{1}} \cdots \frac{\pi_{n}}{p_{n}} F\left(p_{1}, \ldots, p_{n}\right)^{l} .
$$



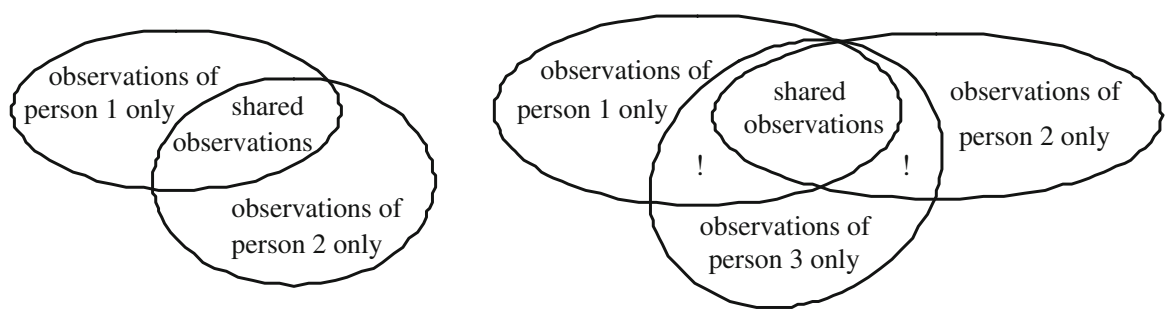

Fig. 1 Observation sets in a group of $n=2$ perons (no subgroup information), and a group of $n=3$ persons (with subgroup information marked by '!')

Relations (11) and (12) together immediately imply that $G$ is externally Bayesian if and only if $F$ is externally Bayesian. Further, again by (9) and (10),

$$
\begin{aligned}
G\left(p_{1}, \pi_{1}, \ldots, p_{i-1}, \pi_{i-1}, p_{i}, \pi_{i}^{l}, p_{i+1}, \pi_{i+1}, \ldots, p_{n}, \pi_{n}\right) & \propto l \frac{\pi_{1}}{p_{1}} \ldots \frac{\pi_{n}}{p_{n}} F\left(p_{1}, \ldots, p_{n}\right) \\
& \propto \frac{\pi_{1}}{p_{1}} \cdots \frac{\pi_{n}}{p_{n}} F\left(p_{1}, \ldots, p_{n}\right)^{l} .
\end{aligned}
$$

This together with (11) implies that $G$ is internally Bayesian if and only it $F$ is externally Bayesian.

\section{When is information independent, when not?}

Let us go back to Theorem 1's assumption of Independent Information (Ind). This assumption is often a useful idealisation, even in situations where it fails. But what exactly are these real situations where (Ind) fails? An important source for failure is what I call subgroup information, that is, information held by more than one but less than all persons. I will prove that, under certain conditions, (Ind) holds if and only if there is no subgroup information.

By a person $i$ 's observation set I mean, informally, the (possibly quite enormous) collection of $i$ 's relevant observations/items of information. Formally, one may define $i$ 's observation set as a set $\mathcal{O}_{i}$ of non-empty observations $O \subseteq \Omega .{ }^{16}$ In the case of a jury faced with hypotheses about the defendant's guilt, $i$ 's observation set might include the observations 'an insecure smile on the defendant's face', 'the defendant's fingerprint near the crime scene', 'two contradictory statements by witness x', etc.

Figure 1 shows observation sets, not sets of possible worlds $A \subseteq \Omega$. These two concepts are in fact opposed to each other: the larger the observation set, the smaller the corresponding set of worlds (in which the observations hold); the union of observation sets compares to the intersection of the sets of worlds. Formally, to an observation set $\mathcal{O}$ corresponds the set of worlds $\cap_{O \in \mathcal{O}} O \subseteq \Omega$ (interpreted as $\Omega$ if $\mathcal{O}=\varnothing$ ). Thus, $i$ 's

\footnotetext{
16 An observation made by every person is represented by the sure event $O=\Omega$, because $\Omega$ is interpreted as containing the worlds that are possible under shared information. Formally, $O \in \mathcal{O}_{1} \cap \ldots \cap \mathcal{O}_{n}$ implies $O=\Omega$.
} 
information $E_{i}$ equals

$$
E_{i}=\bigcap_{O \in \mathcal{O}_{i} \backslash\left(\mathcal{O}_{1} \cap \ldots \cap \mathcal{O}_{n}\right)} O,
$$

the intersection of all of $i$ 's observations except from any shared one; by footnote 16, this actually reduces to

$$
E_{i}=\bigcap_{O \in \mathcal{O}_{i}} O
$$

Here is the problem. Consider any observation contained in the observation sets of more than one but less than all persons $i$-something impossible in groups of size $n=2$ but possible in larger groups, as illustrated by the '!' fields in Fig. 1. This observation is not part of the shared information, but of the personal information $E_{i}$ of many individuals $i$. Such subgroup information typically creates positive correlations between the $E_{i} \mathrm{~s}$ in question. As a stylised example, consider a jury of $n=3$ jurors faced with the hypothesis of guilt of the defendant $(H)$. All jurors have read the charge (shared information), and moreover juror 1 has listened to the first witness report and observed the defendant's nervousness $\left(E_{1}\right)$, juror 2 has listened to the second witness report and observed the defendant's smiles $\left(E_{2}\right)$, and juror 3 has listened to both witness reports and had a private chat with the defendant $\left(E_{3}\right)$. Note the subgroup information of jurors 1 and 3 , and that of jurors 2 and 3 , which typically causes $E_{3}$ to be positively correlated with $E_{1}$ and with $E_{2}$. By contrast, individuals 1 and 2 together have no subgroup information. This situation is depicted in Fig. 1 on the right.

To formally clarify the relationship between subgroup information and independence violation, some preparation is needed.

Definition 4 A subgroup is a non-empty subset $M$ of the group $N:=\{1, \ldots, n\}$. A subgroup is proper if it contains more than one but less than all persons.

To formalise the notion of subgroup information, suppose that to each subgroup $M$ there is a non-empty event $E^{M} \subseteq \Omega, M$ 's exclusively shared information, representing all information held by each of and only the persons in $M$, where by assumption:

- $\quad E_{i}=\bigcap_{\{i\} \subseteq M \subseteq N} E^{M}$ for all persons $i$ (as $i$ has observed those $E^{M}$ with $\left.i \in M\right)^{17}$;

- $\quad E^{N}=\Omega$ (as any world $\omega \in \Omega$ is assumed possible under the shared information);

- each $E^{M}$ belongs to $\mathcal{A}$, the domain of the probability measure $P$ (which holds in particular if $\mathcal{A}$ contains all subsets of $\Omega$ ).

For instance, the '!' fields in Fig. 1 (right) represent the observation sets corresponding to $E^{\{1,3\}}$ and $E^{\{2,3\}} \cdot E^{M}$ is interpretable as the intersection

$$
\bigcap_{O \in\left(\cap_{i \in M} \mathcal{O}_{i}\right) \backslash\left(\cup_{i \notin M} \mathcal{O}_{i}\right)} O
$$

$\overline{17 \text { Why not rather assume that }} E_{i}=\bigcap_{\{i\} \subseteq M \subsetneq N} E^{M}$, as $E_{i}$ should not contain information held by everybody? In fact, both assumption are equivalent since by $E^{N}=\Omega$ an additional intersection with $E^{N}$ has no effect. 
of all observations $O$ contained in each of the observation sets $\mathcal{O}_{i}, i \in M$, but in none of the observation sets $\mathcal{O}_{i}, i \notin M$ (where this intersection is $\Omega$ if $\left(\cap_{i \in M} \mathcal{O}_{i}\right) \backslash\left(\cup_{i \notin M} \mathcal{O}_{i}\right)=$ $\varnothing)$.

What we have to exclude is that a proper subgroup $M$ exclusively shares information; in other words, $E^{M}$ must be the no-information event $\Omega$ :

No Subgroup Information (NoSI) Every proper subgroup $M$ has no exclusively shared information, i.e. $E^{M}=\Omega$ (or, more generally, $P\left(E^{M}\right)=1^{18}$ ).

This condition is empty if there are just $n=2$ individuals, it requires $E^{\{1,2\}}=$ $E^{\{1,3\}}=E^{\{2,3\}}=\Omega$ if $n=3$, and it requires the '!' fields in Fig. 1 to be empty. Finally, consider the following independence assumption:

(Ind*) The events $E^{M}, \varnothing \neq M \subseteq N$, are $(P$-)independent conditional on each $H \in \mathcal{H}$.

(Ind*) is a more generally acceptable condition than (Ind) in that the $E^{M} \mathrm{~s}$, unlike the $E_{i} \mathrm{~s}$, are based on non-overlapping observation sets. Indeed, a subgroup $M$ 's exclusively shared information $E^{M}$, by the very meaning of 'exclusively', represents different observations than any other subgroup's exclusively shared information. ${ }^{19}$

Theorem 3 Assume (Ind*). Then:

(a) Independent Information (Ind) is equivalent to No Subgroup Information (NoSI);

(b) specifically, if $E^{M} \neq \Omega$ for proper subgroup $M$, then conditional on at least one $H \in \mathcal{H}$ the personal observations $E_{i}, i \in M$, are pairwise positively correlated (i.e. $P\left(E_{i} \cap E_{j} \mid H\right)>P\left(E_{i} \mid H\right) P\left(E_{j} \mid H\right)$ for any two distinct $\left.i, j \in M\right)$.

Proof Suppose (Ind*). I prove part (a); the proof includes a proof of part (b).

(i) First, assume (NoSI). Each event $E^{\{i\}}$ coincides with $E_{i}$ up to a set of probability zero, because

$$
E_{i}=\bigcap_{\{i\} \subseteq M \subseteq N} E^{M}=E^{\{i\}} \bigcap\left(\bigcap_{\{i\} \subseteq M \subseteq N \&|M| \geq 2} E^{M}\right),
$$

in which $P\left(\bigcap_{\{i\} \subseteq M \subseteq N \&|M| \geq 2} E^{M}\right)=1$ by (NoSI). So, as the events $E^{\{1\}}, \ldots, E^{\{n\}}$ are independent conditional on any $H \in \mathcal{H}$ by (Ind*), also the events $E_{1}, \ldots, E_{n}$ are independent conditional on any $H \in \mathcal{H}$.

(ii) Now assume (NoSI) is violated, and let $M^{*}$ be a proper subgroup with $P\left(E^{M^{*}}\right)<1$. I show that the events $E_{i}, i \in M^{*}$, are pairwise positively

$\overline{18}$ ' $P\left(E^{M}\right)=1$ ' is equivalent to ' $E M=\Omega$ ' in the natural case that only the empty event in $\mathcal{A}$ has zero probability. Strictly speaking, ' $E^{M}=\Omega$ ' means 'no information' while ' $P\left(E^{M}\right)=1$ ' means 'essentially no information'. I am grateful to the referee for suggesting to require ' $P\left(E^{M}\right)=1$ ' instead of ' $E^{M}=\Omega$ ', thereby making it possible to state Theorem 3 without assuming that only the empty event in $\mathcal{A}$ has zero probability.

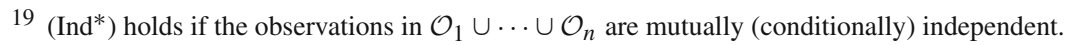


correlated conditional on at least one $H \in \mathcal{H}$ (which proves part (b) and also completes the proof of part (a) since $E_{1}, \ldots, E_{n}$ are then not independent conditional on that $H)$. Consider any distinct $i, j \in M^{*}$. By $P\left(E^{M^{*}}\right)<1$ there exists an $H \in \mathcal{H}$ with $P\left(E^{M^{*}} \mid H\right)<1$. Since $E_{i}=\bigcap_{\{i\} \subseteq M \subseteq N} E^{M}$ and using (Ind*), we have

$$
P\left(E_{i} \mid H\right)=\prod_{\{i\} \subseteq M \subseteq N} P\left(E^{M} \mid H\right)
$$

The analogous argument for $j$ yields

$$
P\left(E_{j} \mid H\right)=\prod_{\{j\} \subseteq M \subseteq N} P\left(E^{M} \mid H\right) .
$$

So,

$$
P\left(E_{i} \mid H\right) P\left(E_{j} \mid H\right)=\left[\prod_{\{i\} \subseteq M \subseteq N} P\left(E^{M} \mid H\right)\right] \times\left[\prod_{\{j\} \subseteq M \subseteq N} P\left(E^{M} \mid H\right)\right] .
$$

Further, we have

$$
\begin{aligned}
E_{i} \cap E_{j} & =\left[\bigcap_{\{i\} \subseteq M \subseteq N} E^{M}\right] \bigcap\left[\bigcap_{\{j\} \subseteq M \subseteq N} E^{M}\right] \\
& =\left[\bigcap_{\{i\} \subseteq M \subseteq N} E^{M}\right] \bigcap\left[\bigcap_{\{j\} \subseteq M \subseteq N \backslash\{i\}} E^{M}\right] .
\end{aligned}
$$

So, by (Ind*),

$$
P\left(E_{i} \cap E_{j} \mid H\right)=\left[\prod_{\{i\} \subseteq M \subseteq N} P\left(E^{M} \mid H\right)\right] \times\left[\prod_{\{j\} \subseteq M \subseteq N \backslash\{i\}} P\left(E^{M} \mid H\right)\right] .
$$

The relations (13) and (14) together entail

$$
P\left(E_{i} \cap E_{j} \mid H\right)>P\left(E_{i} \mid H\right) P\left(E_{j} \mid H\right),
$$

because expression (13) equals expression (14) multiplied with the factor

$$
\prod_{\{i, j\} \subseteq M \subseteq N} P\left(E^{M} \mid H\right)
$$


which is smaller than 1 since it contains the term $P\left(E^{M^{*}} \mid H\right)<1$.

\section{Opinion pooling in the presence of subgroup information}

One may always try to 'remove' subgroup information through active information sharing prior to aggregation: all proper subgroups with exclusively shared information communicate this information to the rest of the group. In Fig. 1, the observations in each '!' field are communicated to the third person, and in the above jury example the subgroups $\{1,3\}$ and $\{2,3\}$ communicate the exact content of the first resp. second witness report to the third juror. Having thus removed any subgroup information, (NoSI) and hence (in view of Theorem 3) Independent Information (Ind) hold, so that opinion pooling can proceed along the lines of Sects. 2-5.

But suppose now that such information sharing is not feasible, e.g. due to the complexity of subgroup information. Then (NoSI) fails, and hence (Ind) fails, so that we need to modify our pooling formula. It is at first not obvious whether and how one can generalise Theorem 1 to arbitrary information overlaps, i.e. whether and how collective opinions can incorporate all information spread around the group. The generalisation is possible, as will be seen. Roughly speaking, we have to replace Theorem 1's axioms of Individual Bayesian Rationality (IBR) and Independent Information (Ind) by corresponding axioms based on subgroups rather than individuals. Theorem 1's two other axioms, (APLA) and (CP), will not anymore appear explicitly, but are build implicitly into the model, as explained in a moment. The adapted axioms will again lead to a unique collective opinion $\pi$, calculated in a somewhat more complicated way than in Theorem 1.

First, let me state the new model ingredients, and compare them to the earlier ingredients. As before, we have a non-empty set of possible worlds $\Omega$, partitioned into a countable set $\mathcal{H}$ of non-empty hypotheses $H$. While Theorem 1 's model contained for every individual $i$ a personal information $E_{i} \subseteq \Omega$, now for every subgroup $M$ there is a non-empty event $E^{M} \subseteq \Omega, M$ 's exclusively shared information, representing all information held by each of and only the persons in $M$. By assumption, $E^{N}=\Omega$, reflecting that any world $\omega \in \Omega$ is possible under the shared information. From these events $E^{M}$ we can define each individual $i$ 's information as

$$
E_{i}=\bigcap_{\{i\} \subseteq M \subseteq N} E^{M},
$$

representing all information held at least by person $i$.

The earlier model contained every individual $i$ 's (prior) belief $P_{i}$; this is not anymore needed here. Instead, I only assume a single probability measure $P$, defined on some $\sigma$-algebra $\mathcal{A} \subseteq \mathcal{P}(\Omega)$ containing each $E^{M}$ and each hypothesis $H \in \mathcal{H}$. We interpret $P$ as capturing common prior beliefs. ${ }^{20}$ This assumption of common prior

\footnotetext{
20 More precisely, I do not mean to assume that every individual $i$ holds a belief on all events in $\mathcal{A}$. Rather $i$ holds beliefs (at least) on a sub- $\sigma$-algebra of $\mathcal{A}$ containing all hypotheses in $\mathcal{H}$ and those events $E^{M}$ for which $i \in M$. $i$ 's beliefs on this sub- $\sigma$-algebra are given by $P$.
} 
beliefs is a simplification; it for instance implies that conditions such as (CP) and (APLA) above are built into the model, and hence will not have to appear explicitly.

Recall further that in Theorem 1's model (in its common prior version) people provide individual opinions $\pi_{1}, \ldots, \pi_{n}$ (reflecting 'individually shared' information) and a common prior opinion $p$ (reflecting the group's shared information). So, technically, the earlier model contained the opinions $\pi_{1}, \ldots, \pi_{n}, p$ reflecting the shared information of the improper subgroups $\{1\}, \ldots,\{n\}, N$, respectively. Our new model adds to this the opinions reflecting the shared information of certain proper subgroups $M \subseteq N$. More precisely, in the new model at least those (proper or improper) subgroup which exclusively share information will need to provide an opinion. Formally, let $\mathcal{M}$ be a set of subgroups, containing at least those (proper or improper) subgroups $M \subseteq N$ with exclusively shared information, i.e. with $E^{M} \neq \Omega$. Without loss of generality, let $N \in \mathcal{M}^{21}$ Each subgroup $M$ in $\mathcal{M}$ submits an opinion $\pi_{M} \in \Pi$, representing $M$ 's probability assignments based on $M$ 's shared information (shared information need not be exclusively shared, i.e. may be known to other persons too; see Definition 5 below). Theorem 1's model (in the common prior version) is the special case that $\mathcal{M}=\{\{1\}, \ldots,\{n\}, N\}(=\{M: M$ is an improper subgroup $\})$ with $\pi_{\{1\}}=\pi_{1}, \ldots, \pi_{\{n\}}=\pi_{n}, \pi_{N}=p$. In the last section's jury example with $n=3$ individuals, we may put

$$
\mathcal{M}=\{\{1\},\{2\},\{3\},\{1,3\},\{2,3\},\{1,2,3\}\}
$$

because $\{1,2\}$ has no exclusively shared information.

In practice, in addition to every individual $i$ with $\{i\} \in \mathcal{M}$ submitting an opinion $\pi_{\{i\}}$, every non-singleton subgroup $M \in \mathcal{M}$ will have to 'sit together', find out about the information it shares, and come up with an opinion $\pi_{M}$ based on this shared information.

The technique to calculate the (collective) opinion $\pi \in \Pi$ from the submitted subgroup opinions $\pi_{M}, M \in \mathcal{M}$, is recursive. Let me first illustrate it by an example.

Example 1 As in the last section's jury example, let there be $n=3$ individuals and let $\mathcal{M}=\{\{1\},\{2\},\{3\},\{1,3\},\{2,3\},\{1,2,3\}\}$. So, functions $\pi_{\{1\}}, \pi_{\{2\}}, \pi_{\{3\}}, \pi_{\{1,3\}}, \pi_{\{2,3\}}$ and $\pi_{\{1,2,3\}}$ are submitted. The recursion works as follows, where I use a slightly simplified version of the later notation and give only informal justifications.

- First, merge $\pi_{\{1,3\}}$ and $\pi_{\{2,3\}}$ into a function $\pi_{\{1,3\},\{2,3\}}$ that combines $\{1,3\}$ 's shared information and $\{2,3\}$ 's shared information. One may apply Corollary 1's formula:

$$
\pi_{\{1,3\},\{2,3\}} \propto \pi_{\{1,3\}} \pi_{\{2,3\}} / \pi_{\{1,2,3\}} .
$$

(To see why $\pi_{\{1,2,3\}}$ can play the role of the prior opinion $p$ in Corollary 1 , recall that $p$ there represents the information shared by all individual opinions. The

\footnotetext{
${ }^{21}$ One may always define $\mathcal{M}$ as containing all subgroups, but in practice this maximal choice adds unnecessary steps to the recursive pooling procedure introduced below. The minimal choice is $\mathcal{M}=\{M: \emptyset \neq$ $M \subsetneq N$ and $\left.E^{M} \neq \Omega\right\} \cup\{N\}$.
} 
information shared by the opinions $\pi_{\{1,3\}}$ and $\pi_{\{2,3\}}$ is the information held by [1 and 3 ] and by [2 and 3]. This is equivalent to the information held by 1 and 2 and 3 , i.e. the information expressed in $\pi_{\{1,2,3\}}$.)

- Next, define $\pi_{\{1,2\}}$ as $\pi_{\{1,2,3\}}$, because the subgroup $\{1,2\}$ does not exclusively share any information and hence shares the same information as the larger group $\{1,2,3\}$.

- Next, merge $\pi_{\{1\}}$ and $\pi_{\{2\}}$ into a function $\pi_{\{1\},\{2\}}$ that combines $\{1\}$ 's and $\{2\}$ 's information. One may apply Corollary 1's formula:

$$
\pi_{\{1\},\{2\}} \propto \pi_{\{1\}} \pi_{\{2\}} / \pi_{\{1,2\}} .
$$

(Why can $\pi_{\{1,2\}}$ play the role of $p$ in Corollary 1 , i.e. why does $\pi_{\{1,2\}}$ express the information shared by $\pi_{\{1\}}$ and $\pi_{\{2\}}$ ? The information shared by $\pi_{\{1\}}$ and $\pi_{\{3\}}$ is the information held by 1 and by 2 , i.e. the information expressed in $\pi_{\{1,2\}}$.)

- Finally, merge $\pi_{\{1\},\{2\}}$ and $\pi_{\{3\}}$ into the function $\pi=\pi_{\{1\},\{2\},\{3\}}$ that combines $\{1\}$ 's, $\{2\}$ 's and $\{3\}$ 's information. Again, one may apply Corollary 1's formula:

$$
\pi=\pi_{\{1\},\{2\},\{3\}} \propto \pi_{\{1\},\{2\}} \pi_{\{3\}} / \pi_{\{1,3\},\{2,3\}} .
$$

(Why can $\pi_{\{1,3\},\{2,3\}}$ play the role of $p$ in Corollary 1, i.e. why does $\pi_{\{1,3\},\{2,3\}}$ represent the information shared by $\pi_{\{1\},\{2\}}$ and $\pi_{\{3\}}$ ? The information shared by $\pi_{\{1\},\{2\}}$ and $\pi_{\{3\}}$ is the information held by [1 or 2] and by 3 . This is precisely the information held by [1 and 3] or by [2 and 3], i.e. the information expressed in $\pi_{\{1,3\},\{2,3\} .)}$

Now I come to the formal treatment. Recall that $i$ 's information $E_{i}$ is given by

$$
E_{i}=\bigcap_{\{i\} \subseteq M \subseteq N} E^{M},
$$

i.e. $i$ knows precisely the conjunction of what the subgroups containing $i$ exclusively share. This generalises as follows to:

Definition 5 A subgroup M's shared information is defined as

$$
E_{M}:=\bigcap_{M \subseteq M^{\prime} \subseteq N} E^{M^{\prime}}
$$

(the conjunction of all information exclusively shared by some supergroup of $M$ ).

$E_{M}$ represents what is known to at least all members of $M$ - as opposed to $M$ 's exclusively shared information $E^{M}$, known exactly all members of $M$. Taking the case of a singleton subgroup $M=\{i\}$, the event $E_{\{i\}}$ coincides with $E_{i}$. Also, note that

$$
P\left(E^{M}\right)>0 \text { and } P\left(E_{M}\right)>0 \text { for each subgroup } M
$$


because

$$
P\left(E^{M}\right), P\left(E_{M}\right) \geq P\left(\bigcap_{\varnothing \neq M^{\prime} \subseteq N} E^{M^{\prime}}\right)=P\left(E_{1} \cap \ldots \cap E_{n}\right)>0 .
$$

The following condition translates Individual Bayesian Rationality (IBR) to subgroups in $\mathcal{M}$ :

Subgroup Bayesian Rationality (SBR) $\pi_{M}(H)=P\left(H \mid E_{M}\right)$ for every subgroup $M \in \mathcal{M}$ and hypothesis $H \in \mathcal{H}$.

As in Theorem 1, we would like the collective opinion to satisfy Collective Bayesian Rationality (CBR); that is, we require that

$$
\pi(H)=P\left(H \mid E_{1} \cap \cdots \cap E_{n}\right) \text { for each hypothesis } H \in \mathcal{H},
$$

a condition that may be rewritten in several equivalent ways since (by Definition 5)

$$
E_{1} \cap \cdots \cap E_{n}=E_{\{1\}} \cap \cdots \cap E_{\{n\}}=\bigcap_{\varnothing \neq M \subseteq N} E^{M}=\bigcap_{\varnothing \neq M \subseteq N} E_{M} .
$$

As a technical tool to construct collective opinion $\pi$ satisfying (CBR), I need to introduce opinions of abstract individuals.

Definition 6 An abstract individual is a non-empty set $A$ of subgroups $M$; its order is $\operatorname{order}(A):=\min \{|M|: M \in A\}$, the size of a smallest subgroup in $A$.

The opinions $\pi_{\{1,3\},\{2,3\}}, \pi_{\{1\},\{2\}}, \ldots$ defined in the example above are in fact the opinions of the abstract individuals $\{\{1,3\},\{2,3\}\},\{\{1\},\{2\}\}, \ldots$ More generally, I interpret an abstract individual $A$ as a hypothetical agent who knows the shared information of any subgroup $M \in A$ (and no more). For instance, $A=\{\{1,3\},\{2,3\}\}$ knows $\{1,3\}$ 's shared information and $\{2,3\}$ 's shared information. $A$ 's information is thus given by $\bigcap_{M \in A} E_{M}$. To get a concrete idea, note that the abstract agent $A=$ $\{\{1,3\},\{2,3\}\}$ knows

- at least as much as the abstract agent $\{\{1,3\}\}$, who knows all information that 1 and 3 share (but no information that 2 and 3 share exclusively);

- at least as much as the abstract agent $\{\{1,2,3\}\}$, who knows all information that 1, 2 and 3 share (but no information that 1 and 3 share exclusively or that 2 and 3 share exclusively);

- at most as much as the abstract agent $\{\{1\},\{2\},\{3\}\}$, who knows all that 1 or 2 or 3 knows (and hence all that two or three of these individuals know together).

I will calculate for each abstract individual $A$ an opinion $\pi_{A} \in \Pi$ that reflects precisely $A$ 's information $\cap_{M \in A} E_{M}$, i.e. that satisfies

$$
\pi_{A}(H)=P\left(H \mid \bigcap_{M \in A} E_{M}\right) \text { for each } H \in \mathcal{H}
$$


Specifically, I calculate $\pi_{A}$ by backward recursion over $\operatorname{order}(A): \pi_{A}$ is calculated first for $\operatorname{order}(A)=n$, then for $\operatorname{order}(A)=n-1, \ldots$, then for $\operatorname{order}(A)=1$. This finally yields $\pi$, since by (CBR) and (15)

$$
\pi=P\left(. \mid E_{\{1\}} \cap \ldots \cap E_{\{n\}}\right)=\pi_{A}
$$

where $A$ is the abstract individual $\{\{1\},\{2\}, \ldots,\{n\}\}$ of order 1 . In the recursive construction, the main steps are to calculate from opinions $\pi_{A}$ and $\pi_{A^{*}}$ of abstract individuals $A$ and $A^{*}$ the opinion $\pi_{A \cup A^{*}}$ of the abstract individual $A \cup A^{*}$ whose information combines the information of $A$ and $A^{*}$. To derive $\pi_{A \cup A^{*}}$ from $\pi_{A}$ and $\pi_{A^{*}}$, I generalise the formula of Theorem 1 to (two) abstract individuals. To do so, the notion of shared information is crucial. What information do $A$ and $A^{*}$ share? They share precisely the information held by the abstract individual

$$
A \vee A^{*}:=\left\{M \cup M^{*}: M \in A \text { and } M^{*} \in A^{*}\right\}
$$

The reason is: the information $A$ and $A^{*}$ share is precisely the information that $A$ knows and $A^{*}$ knows, i.e. that some subgroup in $A$ shares and some subgroup in $A^{*}$ shares, i.e. that some union $M \cup M^{*}$ with $M \in A$ and $M^{*} \in A^{*}$ shares. So, when combining opinions $\pi_{A}$ and $\pi_{A^{*}}, A \vee A^{*}$ 's opinion $\pi_{A \vee A^{*}}$ plays the role of the common prior $p$ in Theorem 1. More precisely, the crucial result on how to combine opinions of abstract individuals states as follows (and is proved later):

Lemma 1 Assume (Ind ${ }^{*}$ ). Consider abstract individuals $B$ and $C$, form the abstract individuals $B \vee C$ and $B \cup C$. If $\pi_{B}, \pi_{C}, \pi_{B \vee C}$ are opinions in $\Pi$ given by (15), then

- there is an opinion in $\Pi$ proportional to $\pi_{B} \pi_{C} / \pi_{B \vee C},{ }^{22}$

- this opinion is the function $\pi_{B \cup C}$ given by (15).

The formula in Lemma 1 guides us in assigning opinions to abstract individuals. The assignment is recursive, with another nested recursion in 'Case 2':

Definition 7 Define the opinions $\pi_{A} \in \Pi$ of abstract individual $A$ by the following backward recursion on $\operatorname{order}(A)$ :

- Assume $\operatorname{order}(A)=n$. Then $A=\{N\}$. Define $\pi_{A}:=\pi_{N}$.

- Assume $\operatorname{order}(A)=k<n$ and assume $\pi_{A^{\prime}}$ is already defined for $\operatorname{order}\left(A^{\prime}\right)>k$. Case 1: $|A|=1$. Then $A=\{M\}$. If $M \in \mathcal{M}$, define $\pi_{A}=\pi_{M}$. If $M \notin \mathcal{M}$, consider the abstract individual $A^{\prime}:=\{M \cup\{i\}: i \notin M\}$ containing all subgroups with exactly one person added to $M$ (interpretation: $A$ and $A^{\prime}$ have the same information by $M \notin \mathcal{M}$ ) and define $\pi_{A}:=\pi_{A^{\prime}}$ (where $\pi_{A^{\prime}}$ is already defined by $\left.\operatorname{order}\left(A^{\prime}\right)=k+1\right)$.

Case 2: $|A|>1$. Define $\pi_{A}$ by another recursion on $|\{M \in A:|M|=k\}|$, the number of subgroups in $A$ of size $k$ :

22 Equivalently, the sum $\sum_{H \in \mathcal{H}} \pi_{B}(H) \pi_{C}(H) / \pi_{B \vee C}(H)$ is finite. Indeed, a function $f$ from $\mathcal{H}$ to $(0, \infty)$ (such as $\pi_{B} \pi_{C} / \pi_{B \vee C}$ ) can be normalised to a function with sum 1 if and only if $f$ has a finite sum. 
- Assume $|\{M \in A:|M|=k\}|=1$. Then $A=\{M\} \cup A^{*}$, where $|M|=k$ and $\operatorname{order}\left(A^{*}\right)>k$. Define $\pi_{A}$ by $\pi_{A} \propto \pi_{\{M\}} \pi_{A^{*}} / \pi_{\{M\} \vee A^{*}}$ (where $\pi_{\{M\}}$ is already defined in case 1 , and $\pi_{A^{*}}$ and $\pi_{\{M\} \vee A^{*}}$ are already defined by $\operatorname{order}\left(A^{*}\right)>k$ and $\left.\operatorname{order}\left(\{M\} \vee A^{*}\right)>k\right)$.

- Assume $|\{M \in A:|M|=k\}|=l>1$ and assume $\pi_{A^{*}}$ is already defined for all the $A^{*}$ such that $\left|\left\{M \in A^{*}:|M|=k\right\}\right|<l$ (and $\operatorname{order}\left(A^{*}\right)=k$ ). Then $A=\{M\} \cup A^{*}$ with $|M|=k$ and $\left|\left\{M^{*} \in A^{*}:\left|M^{*}\right|=k\right\}\right|=l-1$. Define $\pi_{A}$ by $\pi_{A} \propto \pi_{\{M\}} \pi_{A^{*}} / \pi_{\{M\} \vee A^{*}}$ (where $\pi_{\{M\}}$ is already defined in case $1, \pi_{A^{*}}$ is already defined by $\left|\left\{M^{*} \in A^{*}:\left|M^{*}\right|=k\right\}\right|=l-1$, and $\pi_{\{M\} \vee A^{*}}$ is already defined by $\left.\operatorname{order}\left(\{M\} \vee A^{*}\right)>k\right)$.

On the last recursion step we reach the opinions $\pi_{A}$ of abstract individuals of order 1 , hence in particular the opinion of $A=\{\{1\}, \ldots,\{n\}\}$, and this is the desired opinion that incorporates the group's full information:

Theorem 4 If subgroups satisfy (SBR), information satisfies (Ind*), and the collective


calculated) opinion of the abstract individual $\{\{1\}, \ldots,\{n\}\}$.

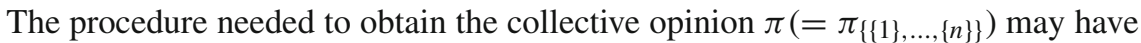
a high complexity. ${ }^{23}$ How practically feasible is it? One should distinguish two separate tasks: (i) first, each subgroup $M$ in $\mathcal{M}$ has to form and submit an opinion $\pi_{M}$; (ii) subsequently, the collective opinion $\pi$ has to be derived algorithmically from the various subgroup opinions. Let me comment on each task.

Whether task (i) is feasible in practice depends crucially on the number and size of subgroups in $\mathcal{M}$, which in turn depends on how information is distributed across people. In the worst case, every subgroup exclusively shares information. Here, $\mathcal{M}=$ $\mathcal{P}(N) \backslash\{\varnothing\}$ and $|\mathcal{M}|=2^{n}-1$, and the task becomes infeasible already for moderately large $n$. On the other hand, the task seems more feasible in situations where only relatively few subgroups exclusively share information. Suppose for instance that, when pooling expert opinions relative to certain hypotheses about climate change, only the following subgroups exclusively share information: each single expert, i.e. each singleton subgroup $\{i\} \subseteq N$; a group of physicists $M_{1} \subseteq N$; a group of biologists $M_{2} \subseteq N$; and a group of meteorologists $M_{3} \subseteq N$. Then we may define $\mathcal{M}$ as $\left\{\{1\},\{2\}, \ldots,\{n\}, M_{1}, M_{2}, M_{3}, N\right\}$, so that only $|\mathcal{M}|=n+4$ opinions have to be formed and submitted.

Task (ii) involves an algorithm with a nested recursion; the overall number of steps grows more than exponentially in $n .{ }^{24}$ So, for large $n$, task (ii) poses a feasibility problem-even if $|\mathcal{M}|$ is small, i.e., if task (i) seems feasible. There is however an escape to this problem if $\mathcal{M}$ contains only relatively small proper subgroups. Let $m:=\max _{M \in \mathcal{M} \backslash\{N\}}|M|$ denote the maximal size that subgroups in $\mathcal{M}$ can have (apart from the improper subgroup $N$ ). A quick inspection of the algorithm in Definition 7 shows that its backward recursion (which assigns opinions $\pi_{A}$ to abstract agents $A$ )

\footnotetext{
23 I am grateful to the referee for drawing my attention to this point.

24 In the algorithm, for each abstract agent $A$ an opinion $\pi_{A}$ is calculated. There are $2^{2^{n}-1}-1$ abstract agents in total. Hence, $2^{2^{n}-1}-1$ opinions have to be calculated.
} 
is trivial until it reaches abstract agents of order $m$ : all abstract agents of order $k>m$ get assigned the opinion $\pi_{A}=\pi_{N}$. So a shortcut is possible: define $\pi_{A}$ as $\pi_{N}$ for all abstract agents $A$ with $\operatorname{order}(A)>m$, and start the backward recursion with those abstract agents $A$ with $\operatorname{order}(A)=m$.

Even if both tasks (i) and (ii) turn out to be practically feasible, the very choice of $\mathcal{M}$ (before starting task (i)) may pose another high-complexity problem. Suppose $\mathcal{M}$ is chosen by surveying all subgroups one by one to find out which ones exclusively share information (each subgroup might be asked to 'sit together' and look for potential information overlaps). Since there are $2^{n}-1$ subgroups in total, this would become infeasible already for moderately large $n$. However, no such problem arises if $\mathcal{M}$ can be specified without performing an explicit subgroup-by-subgroup examination. For instance, $\mathcal{M}$ might be specified by a social planner who knows from the start that certain subgroups (say, those containing experts from different fields) do not exclusively share any information, while the other subgroups might exclusively share information.

Turning now to the proof, I first show Lemma 1 and then Theorem 4.

Proof of Lemma 1 Assume (Ind*). Let $B, C$ be abstract individuals, and $\pi_{B}, \pi_{C}$, $\pi_{B \vee C}, \pi_{B \cup C} \in \Pi$. Suppose $\pi_{B}, \pi_{C}, \pi_{B \vee C}$ satisfy (15). For all abstract individuals $A$, put

$$
\bar{A}:=\left\{M \subseteq N: M^{\prime} \subseteq M \text { for some } M^{\prime} \in A\right\},
$$

the set of supergroups of subgroups in $A$. By (15), $\pi_{B \vee C}=P\left(. \mid \bigcap_{M \in B \vee C} E_{M}\right)$, where by Definition 5

$$
\bigcap_{M \in B \vee C} E_{M}=\bigcap_{M \in B \vee C} \bigcap_{M \subseteq M^{\prime} \subseteq N} E^{M^{\prime}}=\bigcap_{M \in \overline{B \vee C}} E^{M} .
$$

So,

$$
\pi_{B \vee C}=P(. \mid E) \text { with } E:=\bigcap_{M \in \overline{B \vee C}} E^{M} .
$$

Analogously, by (15), $\pi_{B}=P\left(. \mid \bigcap_{M \in B} E_{M}\right)$, where by Definition 5

$$
\bigcap_{M \in B} E_{M}=\bigcap_{M \in B} \bigcap_{M \subseteq M^{\prime} \subseteq N} E^{M^{\prime}}=\bigcap_{M \in \bar{B}} E^{M}=E_{B} \cap E
$$

with $E_{B}:=\bigcap_{M \in \bar{B} \backslash \overline{B \vee C}} E^{M}$. So $\pi_{B}=P\left(. \mid E_{B} \cap E\right)$, and hence by Bayes' rule

$$
\pi_{B} \propto P(. \mid E) P\left(E_{B} \mid . \cap E\right) .
$$

By an analogous argument for $C$, we have

$$
\pi_{C} \propto P(. \mid E) P\left(E_{C} \mid . \cap E\right),
$$


where $E_{C}:=\bigcap_{M \in \bar{C} \backslash \overline{B \vee C}} E^{M}$. By (16), (17) and (18) we have

$$
\begin{gathered}
\pi_{B} \pi_{C} / \pi_{B \vee C} \propto\left[P(. \mid E) P\left(E_{B} \mid . \cap E\right)\right]\left[P(. \mid E) P\left(E_{C} \mid . \cap E\right)\right] / P(. \mid E) \\
=P(. \mid E) P\left(E_{B} \mid . \cap E\right) P\left(E_{C} \mid . \cap E\right) .
\end{gathered}
$$

Note that each of $E_{B}, E_{C}, E$ is an intersection of a set of events of type $E^{M}$, where the three sets of $E^{M} \mathrm{~s}$ (corresponding to $E_{B}, E_{C}, E$, respectively) are pairwise disjoint. So, as by (Ind*) all $E^{M}$ s are independent conditional on any $H \in \mathcal{H}$, so are the events $E_{B}, E_{C}, E$. Consider an $H \in \mathcal{H}$. As $E_{B}, E_{C}, E$ are independent given $H$, the events $E_{B}, E_{C}$ are independent given $H \cap E$. So

$$
P\left(E_{B} \mid . \cap E\right) P\left(E_{C} \mid . \cap E\right)=P\left(E_{B} \cap E_{C} \mid \cap E\right) .
$$

Substituting this into (19) and then applying Bayes' rule, we obtain

$$
\pi_{B} \pi_{C} / \pi_{B \vee C} \propto P(. \mid E) P\left(E_{B} \cap E_{C} \mid . \cap E\right) \propto P\left(. \mid E_{B} \cap E_{C} \cap E\right) \in \Pi .
$$

Now suppose $\pi_{B \cup C}=P\left(. \mid E_{B} \cap E_{C} \cap E\right)$. We may rewrite $E_{B} \cap E_{C} \cap E$ as

$$
\bigcap_{M \in \overline{B \cup C}} E^{M}=\bigcap_{M \in B \cup C} \bigcap_{M \subseteq M^{\prime} \subseteq N} E^{M}=\bigcap_{M \in B \cup C} E_{M},
$$

and hence $\pi_{B \cup C}$ equals $P\left(. \mid \bigcap_{M \in B \cup C} E_{M}\right)$, i.e. satisfies (15).

Proof of Theorem 4 Assume (SBR) and (Ind*). By backward induction on the order of $A$ I show that each abstract individual $A$ has opinion $\pi_{A}$ satisfying (15). This in particular implies that $\{\{1\}, \ldots,\{n\}\}$ has opinion

$$
\pi_{\{\{1\}, \ldots,\{n\}\}}(H)=P\left(H \mid E_{1} \cap \ldots \cap E_{n}\right) \quad \text { for each } H \in \mathcal{H},
$$

so that under (CBR) we have $\pi=\pi_{\{\{1\}, \ldots,\{n\}\}}$, as desired.

Denote by $\mathbf{A}$ the set of abstract individuals $A$. The recursion proceeds as follows.

- If $\operatorname{order}(A)=n$, then $A=\{N\}$, and by definition $\pi_{A}=\pi_{N}$. So by (SBR) $\pi_{A}=P\left(. \mid E_{N}\right)=P\left(. \mid \bigcap_{M \in A} E_{M}\right)$, as desired.

- Now let $\operatorname{order}(A)=k<n$, and assume (15) holds for all $A^{\prime} \in \mathbf{A}$ with $\operatorname{order}\left(A^{\prime}\right)>k$. I have to show that $\pi_{A}=P\left(. \mid \bigcap_{M \in A} E_{M}\right)$.

Case 1: $|A|=1$. Then $A=\{M\}$ with $|M|=k$. If $M \in \mathcal{M}$, then by definition $\pi_{A}=\pi_{M}$, so by $(\mathrm{SBR}) \pi_{A}=P\left(. \mid E_{M}\right)=P\left(. \mid \bigcap_{M^{\prime} \in A} E_{M^{\prime}}\right)$, as desired. Now assume $M \notin \mathcal{M}$. Then by definition $\pi_{A}=\pi_{A^{\prime}}$ with $A^{\prime}:=$ $\{M \cup\{i\}: i \notin M\}$. Since $\operatorname{order}\left(A^{\prime}\right)=k+1$, the induction hypothesis yields $\pi_{A^{\prime}}=P\left(. \mid \bigcap_{M^{\prime} \in A^{\prime}} E_{M^{\prime}}\right)$, hence $\pi_{A}=P\left(. \mid \bigcap_{M^{\prime} \in A^{\prime}} E_{M^{\prime}}\right)$. So I have to show that $\bigcap_{M^{\prime} \in A^{\prime}} E_{M^{\prime}}=E_{M}$. By Definition 5,

$$
E_{M}=\bigcap_{M \subseteq M^{\prime} \subseteq N} E^{M^{\prime}}=E^{M} \bigcap\left\{\bigcap_{M^{\prime} \in A^{\prime}}\left[\bigcap_{M^{\prime} \subseteq M^{\prime \prime} \subseteq N} E^{M^{\prime \prime}}\right]\right\} .
$$


In this, $E^{M}=\Omega$ (by $M \notin \mathcal{M}$ ) and $\bigcap_{M^{\prime} \subseteq M^{\prime \prime} \subseteq N} E^{M^{\prime \prime}}=E_{M^{\prime}}$ (by Definition 5). So $E_{M}=\bigcap_{M^{\prime} \in A^{\prime}} E_{M^{\prime}}$, as desired.

Case 2: $|A|>1$. I show $\pi_{A}=P\left(. \mid \bigcap_{M \in A} E_{M}\right)$ by induction on the number $|\{M \in A:|M|=k\}|$ of subgroups in $A$ of size $k$.

- Let $|\{M \in A:|M|=k\}|=1$. Then $A=\{M\} \cup A^{*}$ with $|M|=k$ and $\operatorname{order}\left(A^{*}\right)>k$. Then $\pi_{A}$ was defined as the function in $\Pi$ proportional to $\pi_{\{M\}} \pi_{A^{*}} / \pi_{\{M\} \vee A^{*}}$; let me show that (i) such a function does indeed exists and (ii) satisfies (15), as desired. Now, $\pi_{\{M\}}$ satisfies (15) by Case 1, and $\pi_{A^{*}}$ and

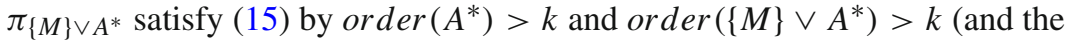
$k$-induction hypothesis). So, by Lemma 1 , the function $\pi_{\{M\}} \pi_{A^{*}} / \pi_{\{M\} \vee A^{*}}$ is proportional to a function in $\Pi$, so that $\pi_{A}$ is well-defined. Also by Lemma 1 , this function $\pi_{A}$ satisfies (15), as desired.

- Let $|\{M \in A:|M|=k\}|=l>1$, and assume $A^{*}$ satisfies (15) whenever $\left|\left\{M \in A^{*}:|M|=k\right\}\right|<l$ (and $\operatorname{order}\left(A^{*}\right)=k$ ). By definition, $\pi_{A} \propto$ $\pi_{\{M\}} \pi_{A^{*}} / \pi_{\{M\} \vee A^{*}}$, where $A=\{M\} \cup A^{*}$ with $|M|=k$ and $\mid\left\{M^{*} \in A^{*}\right.$ : $\left.\left|M^{*}\right|=k\right\} \mid=l-1$. Again, we have to show that $\pi_{A}$ is well-defined (i.e. that $\Pi$ indeed contains a function proportional to $\pi_{\{M\}} \pi_{A^{*}} / \pi_{\{M\} \vee A^{*}}$ ) and satisfies (15). $\pi_{\{M\}}$ satisfies (15) by Case $1, \pi_{A^{*}}$ satisfies (15) by $\mid\left\{M^{*} \in A^{*}:\left|M^{*}\right|=\right.$ $k\} \mid=l-1$ (and the $l$-induction hypothesis), and $\pi_{\{M\} \vee A^{*}}$ satisfies (15) by $\operatorname{order}\left(\{M\} \vee A^{*}\right)>k$ (and the $k$-induction hypothesis). So, by Lemma $1, \pi_{A}$ is well-defined and satisfies (15).

\section{Conclusion}

The above model interprets opinion pooling as information pooling: collective opinions should build in the group's entire information, be it shared or personal. According to the pooling formulae I obtained, collective opinions should account for informational asymmetries not by taking a standard weighted (linear or geometric) average of the individual opinions with higher weight assigned to better informed individuals but by incorporating people's prior opinions in addition to their actual (i.e. posterior) opinions. In practice, people have either to agree on a common prior opinion $p$, i.e. on how to interpret the shared information, or they have to submit their possibly diverging prior opinions $p_{1}, \ldots, p_{n}$. Based on simple axioms, Theorem 1 shows how to aggregate the (prior and posterior) opinions into a collective opinion. The formula defines a multiplicative opinion pool: the collective opinion $\pi$ is the product of the individual opinions $\pi_{1}, \ldots, \pi_{n}$ and a function $g$ (which depends on prior opinions).

More precisely, Theorem 1 suggests that, based on individual opinions $\pi_{1}, \ldots, \pi_{n}$, the collective opinion $\pi$ should be defined by

$$
\pi \propto \pi_{1} \ldots \pi_{n} / p^{n-1}
$$

if people agree on a common prior $p$, and by

$$
\pi \propto \frac{\pi_{1}}{p_{1}} \cdots \frac{\pi_{n}}{p_{n}} F\left(p_{1}, \ldots, p_{n}\right)
$$


if people have arbitrary priors $p_{1}, \ldots, p_{n}$, where $F$ is a standard opinion pool. I have suggested that $F$ should be anonymous (i.e. symmetric in its arguments) because the prior opinions it pools are based on the same (shared) information, giving no individual an informational superiority. More specifically, I have suggested to define $F$ as unweighted geometric pooling, because this generates appealing properties shown in Theorem 2. This choice of $F$ gives collective opinion the form

$$
\pi \propto \frac{\pi_{1}}{p_{1}^{1-1 / n}} \cdots \frac{\pi_{n}}{p_{n}^{1-1 / n}} .
$$

Fortunately, not much depends on how we choose $F$ in the pooling formula (20) if - as is frequently the case $-\frac{\pi_{1}}{p_{1}} \cdots \frac{\pi_{n}}{p_{n}}$ dominates $F\left(p_{1}, \ldots, p_{n}\right)$ (i.e., if the function $\frac{\pi_{1}}{p_{1}} \cdots \frac{\pi_{n}}{p_{n}}$ varies far more than the function $F\left(p_{1}, \ldots, p_{n}\right)$ for 'reasonable' choices of $F$ ). In such cases, one might in practice refrain from choosing $F$ and simply define the collective opinion as

$$
\pi \propto \frac{\pi_{1}}{p_{1}} \cdots \frac{\pi_{n}}{p_{n}}
$$

a particularly elegant pooling formula.

A crucial axiom underlying these pooling formulas is that personal information is independent. By Theorem 3, independence is threatened by the possibility of subgroup information, i.e. of information held by more than one but less than all individuals. Theorem 4 therefore generalises the aggregation rule to arbitrary information distributions (allowing for subgroup information). The generalisation is unique, but assumes that each subgroup with subgroup information agrees on how to interpret this information, a kind of common prior assumption. Dropping this assumption would have gone beyond the scope of this paper, but it might be an interesting route for future research.

Acknowledgments I am very grateful for numerous helpful suggestions by a competent and diligent referee. This paper is based on my old unpublished paper 'Opinion Pooling under Asymmetric Information,' Public Economics 0407002, EconWPA, 2004. Meanwhile, interesting related results have been obtained independently by Marcus Pivato in his working paper 'The Discursive Dilemma and Probabilistic Judgement Aggregation,' MPRA Paper 8412, University Library of Munich, Germany, 2008.

Open Access This article is distributed under the terms of the Creative Commons Attribution Noncommercial License which permits any noncommercial use, distribution, and reproduction in any medium, provided the original author(s) and source are credited.

\section{References}

Broome J (1990) Bolker-Jeffrey expected utility theory and axiomatic utilitarianism. Rev Econ Stud 57:477502

Dietrich F (2004) Opinion pooling under asymmetric information. working paper, Public Economics 0407002, EconWPA

Dietrich F (2008) The premises of Condorcet's jury theorem are not simultaneously justified. Episteme 5(1):56-73 
Dietrich F, List C (2007) Opinion pooling on general agendas. working paper, METEOR Research Memorandum 038, Maastricht University

Fitelson B (2001) A Bayesian account of independent evidence with applications. Phil Sci 68 (Proceedings):S123-S140

Genest C (1984) A characterization theorem for externally Bayesian groups. Ann Stat 12:1100-1105

Genest C, Zidek JV (1986) Combining probability distributions: a critique and an annotated bibliography. Stat Sci 1:114-148

Genest C, McConway KJ, Schervish MJ (1986) Characterization of externally Bayesian pooling operators. Ann Stat 14:487-501

Hild M (1998) The instability of ex post aggregation. Typescript

Hylland A, Zeckhauser R (1979) The impossibility of group decision making with separate aggregation of beliefs and values. Econometrica 47:1321-1336

Jeffrey R (1983) The logic of decision (first published 1965). Chicago University Press, Chicago

Lehrer K, Wagner C (1981) Rational consensus in science and society. Reidel, Dordrecht

Levi I (1990) Pareto-unanimity and consensus. J Philos 87

Madansky A (1964) Externally Bayesian groups. Technical Report RM-4141-PR, RAND Corporation

McConway K (1978) The combination of experts' opinions in probability assessments: some theoretical considerations. Ph.D. thesis, University College London

McConway K (1981) Marginalization and linear opinion pools. J Am Stat Assoc 76:410-414

Mongin P (1995) Consistent Bayesian aggregation. J Econ Theory 66:313-351

Mongin P (1998) The paradox of the Bayesian experts and state-dependent utility theory. J Math Econ 29:331-361

Morris PA (1974) Decision analysis expert use. Manage Sci 20:1233-1241

Pearl J (2000) Causality: models, reasoning and inference. Cambridge University Press, Cambridge

Pivato M (2008) The discursive dilemma and probabilistic judgement aggregation. MPRA Paper 8412, University Library of Munich, Germany

Risse M (2001) Instability of ex post aggregation in the Bolker/Jeffrey framework and related instability phenomena. Erkenntnis 55:239-269

Risse M (2003) Bayesian group agents and two modes of aggregation. Synthese (forthcoming)

Savage L (1954) The foundations of statistics. Wiley, New York

Schervish M, Seidenfeld T, Kadane J (1991) Shared preferences and state-dependent utilities. Manage Sci 37:1575-1589

Seidenfeld T, Kadane J, Schervish M (1989) On the shared preferences of two Bayesian decision makers. J Philos 86:221-244

Wagner CG (1982) Allocation, Lehrer models, and the consensus of probabilities. Theory Decis 14:207-220

Wagner C (1985) On the formal properties of weighted averaging as a method of aggregation. Synthese 62:97-108 